\title{
EFFICIENT STOCHASTIC ASYMPTOTIC-PRESERVING IMPLICIT-EXPLICIT METHODS FOR TRANSPORT EQUATIONS WITH DIFFUSIVE SCALINGS AND RANDOM INPUTS*
}

\author{
SHI JIN ${ }^{\dagger}$, HANQING LU ${ }^{\ddagger}$, AND LORENZO PARESCHI ${ }^{\S}$
}

\begin{abstract}
For linear transport and radiative heat transfer equations with random inputs, we develop new generalized polynomial chaos based asymptotic-preserving stochastic Galerkin schemes that allow efficient computation for the problems that contain both uncertainties and multiple scales. Compared with previous methods for these problems, our new method uses the implicit-explicit time discretization to gain higher order accuracy, and by using a modified diffusion operator based penalty method, a more relaxed stability condition - a hyperbolic, rather than parabolic, CFL stability condition - is achieved in the case of a small mean free path in the diffusive regime. The stochastic asymptotic-preserving property of these methods will be shown asymptotically and demonstrated numerically, along with a computational cost comparison with previous methods.
\end{abstract}

Key words. transport equation, radiative heat transfer, uncertainty quantification, asymptotic preserving, diffusion limit, stochastic Galerkin, implicit-explicit Runge-Kutta methods

AMS subject classifications. 35Q20, 65M70

DOI. $10.1137 / 17 \mathrm{M} 1120518$

1. Introduction. Transport equations, for example, linear transport equations and radiative transfer equations, arise in many important physical applications, from the microscopic neutron transport to large-scale astrophysical problems [10]. As in general kinetic theory, one of the main challenges is its high dimensionality, since these equations describe particle density distributions defined in the phase space, with independent variables time, space, and particle velocity. Another challenge is the multiscale nature, since the Knudsen number, the dimensionless mean free path, could vary in different orders of magnitude in the computational problems. When the Knudsen number is $O(1)$, we are in the kinetic regime. When it is small, usually one can approximate the kinetic equations by hydrodynamic or diffusion type equations which are macroscopic equations defined in physical space and therefore allow more efficient numerical computations. Asymptotic-preserving (AP) schemes, which mimic the asymptotic transition from the kinetic to the macroscopic equation at the discrete level, have been proven to be an effective computational paradigm for such multiscale kinetic or transport equations and have found many applications in kinetic theory $[11,12,13,16]$. For those relevant to the equations to be studied in this article, see,

*Submitted to the journal's Methods and Algorithms for Scientific Computing section March 13, 2017; accepted for publication (in revised form) December 18, 2017; published electronically March $1,2018$.

http://www.siam.org/journals/sisc/40-2/M112051.html

Funding: This work was supported by NSF grants DMS-1522184 and DMS-1107291, RNMS KI-Net, by NSFC grant 91330203, and by the Office of the Vice Chancellor for Research and Graduate Education at the University of Wisconsin, Madison, with funding from the Wisconsin Alumni Research Foundation. This work was partially supported by the research grant GNCS-INDAM 2016,

"Numerical Methods for Uncertainty Quantification in Hyperbolic and Kinetic Equations."

${ }^{\dagger}$ Institute of Natural Sciences, School of Mathematical Science, MOE-LSEC and SHL-MAC, Shanghai Jiao Tong University, Shanghai 200240, China, and Department of Mathematics, University of Wisconsin, Madison, WI 53706 (sjin@wisc.edu). $\mathrm{edu})$.

$\ddagger$ Department of Mathematics, University of Wisconsin, Madison, WI 53706 (hanqing@math.wisc.

$\S$ Department of Mathematics \& Computer Science, University of Ferrara, Ferrara 44121, Italy (lorenzo.pareschi@unife.it). 
for example, in neutron transport equations $[2,6,14,20,22,24,26,27,28,30]$ and nonlinear radiative transfer $[5,23,34]$.

In this article, we are interested in addressing the third challenge in kinetic modeling: the uncertainty for transport and radiative transfer equations. Since kinetic equations often arise from microscopic equations, for example, Newton's second law or particle dynamics, taking mean-field limits [4, 33], one often encounters difficulties in determining precisely or accurately terms such as collision kernels or scattering coefficients. In addition, initial, boundary data or forcing or source terms could also be measured inaccurately. Thus uncertainty is intrinsic in kinetic models that must be studied in order to assess the accuracy and validate and enhance the computational reliability of kinetic modelings.

Although uncertainty quantification has been a popular field of scientific computing in many areas of scientific and engineering research, its study for kinetic equations has been scarce. Only recently one sees some development of efficient numerical methods for kinetic equations with uncertainties $[7,15,18,19,21,36]$. In particular, in [21], the notion of stochastic asymptotic-preserving (sAP) was introduced. For generalized polynomial chaos (gPC) based stochastic Galerkin (SG) methods, which are the methods used in this article to study the uncertainties, it requires that an SG method for kinetic equations with uncertainty, as the Knudsen number goes to zero, becomes an SG method for the macroscopic hydrodynamic or diffusion equations. This is the stochastic extension of the deterministic AP schemes. Moreover, as realized in [21], and subsequently in other kinetic equations [18, 19, 36], under the SG framework, one obtains a vectorized system of deterministic kinetic equations based on which the deterministic AP machinery can be easily utilized to develop sAP schemes for random kinetic equations. The gPC approach for uncertain kinetic equations is natural, and efficient, since kinetic equations typically preserve the regularity of the initial data in the random space $[15,17,18,29]$; thus one indeed obtains spectral accuracy in the random space, and then the sAP property allows one to use Knudsen number independent time step, mesh sizes, and the order of polynomial degrees in the gPC method.

While sAP schemes have been developed for the linear transport equation [17, 21] and radiative heat transfer equations [19] with uncertainties, in this article we aim to improve the accuracy and efficiency of these methods. Since the gPC-SG based numerical methods lead to much larger system of equations (compared to the original kinetic equations which are scalar equations), and the random variables that describe the uncertainties often live in high dimension, more efficient numerical methods can help to significantly reduce the computational cost. To this aim, we will make two improvements. First, we utilize the implicit-explicit (IMEX) Runge-Kutta (RK) time marching methods (see $[2,3,31]$ and the references therein) in order to gain high order of accuracy in time discretization. Second, since these equations, due to high scattering rates, encounter diffusive regimes, a typical AP scheme based on explicit discretization of the convection term needs a parabolic type CFL stability condition $\Delta t=O\left(\Delta x^{2}\right)$, where $\Delta t$ is the time step and $\Delta x$ is the spatial mesh size. A fully implicit scheme will overcome this constraint but will introduce algebraic difficulties when inverting the entire transport operator numerically. We use a diffusion operator penalized method introduced in [2], which allows us to improve the CFL condition to a hyperbolic one $\Delta t=O(\Delta x)$. We modify the approach of [2] by replacing the microscopic probability density distribution in the penalty term by the macroscopic density, which further simplifies the inversion of the implicit terms. 
The rest of the paper is organized as follows. We develop the gPC-SG based IMEX-RK sAP schemes for the random linear transport equation in section 2 and for the random radiative heat transfer equations in section 3 . Numerical tests can be found in section 4 that verify the efficiency and sAP property of these methods. Some final considerations are reported in section 5 .

2. The linear neutron transport equation with random inputs. In this section we consider a one-dimensional linear neutron transport equation with random inputs. The randomness may come from the cross section, initial data, and boundary data. Let $f(t, x, v, z)$ be the probability density distribution of particles at position $x \in D \subset \mathbb{R}$, time $t \in \mathbb{R}^{+}$, and depending on $v \in(-1,1)$, which is the cosine of the angle between the particle velocity and position. $z \in I_{z} \subset \mathbb{R}^{d}(d \geq 1)$ is the random variable with finite support $I_{z}$. Then the one-dimensional transport equation is

$$
\varepsilon \partial_{t} f+v \partial_{x} f=\frac{1}{\varepsilon}\left(\frac{\sigma_{s}}{2} \int_{-1}^{1} f\left(v^{\prime}\right) d v^{\prime}-\sigma f\right)+\varepsilon Q,
$$

where $\sigma=\sigma(x, z)$ is the total cross section and $\sigma_{s}=\sigma_{s}(x, z)$ is the scattering cross section. The absorption coefficient $\sigma_{A}=\sigma_{A}(x, z)$ is defined by $\sigma_{A}=\frac{\sigma-\sigma_{s}}{\varepsilon^{2}}, Q=$ $Q(x, z)$ is the source term, and the small parameter $\varepsilon$ is the Knusden number, the dimensionless mean free path. In what follows $\sigma_{A}$ and $Q$ are neglected since they do not increase any numerical difficulty for the numerical approximations.

The well-known diffusion limit $\varepsilon \rightarrow 0$ of the one-dimensional deterministic transport equation is of the following form $[1,25]$ :

$$
\partial_{t} \rho=\partial_{x}\left(\frac{1}{3 \sigma_{s}} \partial_{x} \rho\right)
$$

where

$$
\rho=\frac{1}{2} \int_{-1}^{1} f d v .
$$

One can understand the diffusion limit of the transport equation with random inputs through the following even-odd decomposition [20]. First write (1) for $v>0$ :

$$
\begin{aligned}
& \varepsilon \partial_{t} f(v)+v \partial_{x} f(v)=\frac{1}{\varepsilon}\left(\frac{\sigma_{s}}{2} \int_{-1}^{1} f\left(v^{\prime}\right) d v^{\prime}-\sigma f(v)\right), \\
& \varepsilon \partial_{t} f(-v)-v \partial_{x} f(-v)=\frac{1}{\varepsilon}\left(\frac{\sigma_{s}}{2} \int_{-1}^{1} f\left(v^{\prime}\right) d v^{\prime}-\sigma f(-v)\right) .
\end{aligned}
$$

Now denote the even and odd parities

$$
\begin{aligned}
r(t, x, v, z) & =\frac{1}{2}(f(t, x, v, z)+f(t, x,-v, z)), \\
j(t, x, v, z) & =\frac{1}{2 \varepsilon}(f(t, x, v, z)-f(t, x,-v, z)) .
\end{aligned}
$$

Then (3) becomes

$$
\begin{aligned}
& \partial_{t} r+v \partial_{x} j=-\frac{\sigma_{s}}{\varepsilon^{2}}(r-\rho), \\
& \partial_{t} j+\frac{1}{\varepsilon^{2}} v \partial_{x} r=-\frac{1}{\varepsilon^{2}} \sigma_{s} j .
\end{aligned}
$$

As $\varepsilon \rightarrow 0^{+},(5)$ yields

$$
r=\rho, \quad j=-\frac{v}{\sigma_{s}(x, z)} \partial_{x} r .
$$


Substituting (6) into the first equation of (5) and integrating over $v \in[0,1]$, one gets the limiting diffusion equation with random inputs (2).

Based on this even-odd decomposition, Jin, Xiu, and Zhu [21] proposed a gPC-SG formulation for the linear transport equation (1) with random cross section $\sigma_{s}(x, z)$. The sAP property is also introduced for this problem and shown both asymptotically and numerically for this framework [21]. See also [17] for a sharp regularity and sAP proof. However, a parabolic CFL condition $\Delta t=O\left(\Delta x^{2}\right)$ has to be satisfied for the fully discretized scheme in (5) since the scheme will become an explicit scheme for the limiting diffusion equation (2) as $\varepsilon$ goes to 0 .

Now we propose an efficient sAP method for system (5) by applying the IMEX-RK scheme [2] to the gPC Galerkin formulation to get rid of the parabolic CFL condition. Our scheme will fasten the algorithm significantly especially for the vectorized system after the Galerkin projection and can be shown to be sAP.

2.1. The gPC-SG formulation. To approximate the solution, we use the gPC expansion via an orthogonal polynomials series. That is, for random variable $z \in \mathbb{R}^{d}$, one seeks

$$
\begin{aligned}
& r(t, x, v, z) \approx r_{N}(t, x, v, z)=\sum_{k=1}^{K} \hat{r}_{k}(t, x, v) \Phi_{k}(z), \\
& j(t, x, v, z) \approx j_{N}(t, x, v, z)=\sum_{k=1}^{K} \hat{j}_{k}(t, x, v) \Phi_{k}(z),
\end{aligned}
$$

where $\left\{\Phi_{k}(z), 1 \leq k \leq K, K=\left(\begin{array}{c}d+N \\ d\end{array}\right)\right\}$ are from $\mathbb{P}_{N}^{d}$, the $d$-variate orthogonal polynomials of degree up to $N \geq 1$, and orthonormal

$$
\int_{I_{z}} \Phi_{i}(z) \Phi_{j}(z) \pi(z) d z=\delta_{i j}, 1 \leq i, j \leq K=\operatorname{dim}\left(\mathbb{P}_{N}^{d}\right) .
$$

Here $\pi(z)$ is the probability density function of $z$ and $\delta_{i, j}$ the Kronecker delta function. For example, if $\pi(z)$ is the uniform distribution, then $\left\{\Phi_{k}(z)\right\}$ are the Legendre polynomials; if $\pi(z)$ is the Gaussian distribution, then $\left\{\Phi_{k}(z)\right\}$ are the Hermite polynomials; if $\pi(z)$ is the Gamma distribution, then $\left\{\Phi_{k}(z)\right\}$ are the Laguerre polynomials. We refer to [35] for the study of different probability density functions with corresponding orthogonal polynomials.

Now one inserts the approximation (7) into the governing equation (5) and enforces the residue to be orthogonal to the polynomial space spanned by $\left\{\Phi_{1}, \ldots, \Phi_{K}\right\}$. Then, one obtains a set of vectorized deterministic equations for $\hat{\mathbf{r}}=\left(\hat{r}_{1}, \ldots, \hat{r}_{K}\right)^{T}$ and $\hat{\mathbf{j}}=\left(\hat{j}_{1}, \ldots, \hat{j}_{K}\right)^{T}$ :

$$
\begin{aligned}
& \partial_{t} \hat{\mathbf{r}}+v \partial_{x} \hat{\mathbf{j}}=-\frac{1}{\varepsilon^{2}} \mathbf{S}(x)(\hat{\mathbf{r}}-\hat{\boldsymbol{\rho}}), \\
& \partial_{t} \hat{\mathbf{j}}+\frac{v}{\varepsilon^{2}} \partial_{x} \hat{\mathbf{r}}=-\frac{1}{\varepsilon^{2}} \mathbf{S}(x) \hat{\mathbf{j}}
\end{aligned}
$$

where

$$
\hat{\boldsymbol{\rho}}(x, t)=\langle\hat{\mathbf{r}}\rangle=\int_{0}^{1} \hat{\mathbf{r}} d v,
$$

and $\mathbf{S}(x)=\left(s_{i j}(x)\right)_{1 \leq i, j \leq K}$ is $K \times K$ symmetric and positive definite matrix with entries

$$
s_{i j}(x)=\int_{I_{z}} \sigma_{s}(x, z) \Phi_{i}(z) \Phi_{j}(z) \pi(z) d z .
$$


As $\varepsilon \rightarrow 0^{+}$,

$$
\hat{\mathbf{r}}=\hat{\boldsymbol{\rho}}, \quad \hat{\mathbf{j}}=-v \mathbf{S}^{-1} \partial_{x} \hat{\mathbf{r}} .
$$

Inserting the above into (9a) and integrating over $v$, one obtains

$$
\partial_{t} \hat{\boldsymbol{\rho}}=\frac{1}{3} \partial_{x}\left[\mathbf{S}^{-1}(x) \partial_{x} \hat{\boldsymbol{\rho}}\right] .
$$

Remark 2.1. If one applies the gPC-SG formulation for the limiting diffusion equation (2) directly, one gets

$$
\partial_{t} \tilde{\boldsymbol{\rho}}=\frac{1}{3} \partial_{x}\left[\boldsymbol{\Sigma}(x) \partial_{x} \tilde{\boldsymbol{\rho}}\right]
$$

where $\boldsymbol{\Sigma}(x)=\left(\Sigma_{i j}(x)\right)_{1 \leq i, j \leq K}$ is a $K \times K$ symmetric and positive definite matrix with entries

$$
\Sigma_{i j}(x)=\int_{I_{z}} \frac{1}{\sigma_{s}(x, z)} \Phi_{i}(z) \Phi_{j}(z) \pi(z) d z .
$$

Though $\tilde{\mathbf{S}}$ and $\mathbf{S}^{-1}$ are different matrices, one can follow Theorem 4.2 in [32] to show that the difference between $\tilde{\mathbf{S}} \partial_{x} \hat{\boldsymbol{\rho}}$ and $\mathbf{S}^{-1} \partial_{x} \hat{\boldsymbol{\rho}}$ in $L_{2}$ norm is spectrally small.

\subsection{An efficient IMEX-RK scheme with an improved CFL condition.}

2.2.1. A new penalization method. Following [2], we consider a penalization approach to avoid the parabolic CFL condition of a standard AP scheme in the diffusion limit. By adding and subtracting the term $\frac{\mu}{3} \partial_{x}\left(\mathbf{S}^{-1} \partial_{x} \hat{\boldsymbol{\rho}}\right)$ in $(9 \mathrm{a})$ and the term $\phi v \partial_{x} \hat{\mathbf{r}}$ in $(9 \mathrm{~b})$, we reformulate the problem into an equivalent form

$$
\begin{aligned}
\partial_{t} \hat{\mathbf{r}} & =\left[-v \partial_{x} \hat{\mathbf{j}}-\frac{\mu}{3} \partial_{x}\left(\mathbf{S}^{-1} \partial_{x} \hat{\boldsymbol{\rho}}\right)\right]+\left[-\frac{1}{\varepsilon^{2}} \mathbf{S}(\hat{\mathbf{r}}-\hat{\boldsymbol{\rho}})+\frac{\mu}{3} \partial_{x}\left(\mathbf{S}^{-1} \partial_{x} \hat{\boldsymbol{\rho}}\right)\right] \\
& =\boldsymbol{\xi}_{1}(\hat{\mathbf{r}}, \hat{\mathbf{j}})+\boldsymbol{\xi}_{2}(\hat{\mathbf{r}}), \\
\partial_{t} \hat{\mathbf{j}} & =-\phi v \partial_{x} \hat{\mathbf{r}}-\frac{1}{\varepsilon^{2}}\left(\mathbf{S} \hat{\mathbf{j}}+\left(1-\varepsilon^{2} \phi\right) v \partial_{x} \hat{\mathbf{r}}\right)=\boldsymbol{\eta}_{1}(\hat{\mathbf{r}})+\boldsymbol{\eta}_{2}(\hat{\mathbf{r}}, \hat{\mathbf{j}}),
\end{aligned}
$$

where

$$
\begin{aligned}
& \boldsymbol{\xi}_{1}(\hat{\mathbf{r}}, \hat{\mathbf{j}})=-v \partial_{x} \hat{\mathbf{j}}-\frac{\mu}{3} \partial_{x}\left(\mathbf{S}^{-1} \partial_{x} \hat{\boldsymbol{\rho}}\right) \\
& \boldsymbol{\xi}_{2}(\hat{\mathbf{r}})=-\frac{1}{\varepsilon^{2}} \mathbf{S}(x)(\hat{\mathbf{r}}-\hat{\boldsymbol{\rho}})+\frac{\mu}{3} \partial_{x}\left(\mathbf{S}^{-1} \partial_{x} \hat{\boldsymbol{\rho}}\right) \\
& \boldsymbol{\eta}_{1}(\hat{\mathbf{r}})=-\phi v \partial_{x} \hat{\mathbf{r}} \\
& \boldsymbol{\eta}_{2}(\hat{\mathbf{r}}, \hat{\mathbf{j}})=-\frac{1}{\varepsilon^{2}}\left(\mathbf{S} \hat{\mathbf{j}}+\left(1-\varepsilon^{2} \phi\right) v \partial_{x} \hat{\mathbf{r}}\right)
\end{aligned}
$$

Here we choose $\mu=\mu(\varepsilon)$ such that

$$
\begin{aligned}
& \lim _{\varepsilon \rightarrow 0} \mu=1, \\
& \mu=0 \text { if } \varepsilon=O(1),
\end{aligned}
$$

and $\phi=\phi(\varepsilon)$ such that

$$
\min \left\{1, \frac{1}{\varepsilon}\right\} \leq \phi \leq \max \left\{1, \frac{1}{\varepsilon}\right\}
$$


One possible choice of $\mu$ is given in (32), whereas the restriction on $\phi$ guarantees the positivity of $\phi(\varepsilon)$ and $\left(1-\varepsilon^{2} \phi(\varepsilon)\right)$ so the problem remains well-posed uniformly in $\varepsilon$. We make the same simple choice of $\phi$ as in [20]:

$$
\phi(\varepsilon)=\min \left\{1, \frac{1}{\varepsilon^{2}}\right\} .
$$

Remark 2.2. Compared to the penalty term $\mu v^{2} \partial_{x}\left(\mathbf{S}^{-1} \partial_{x} \hat{\mathbf{r}}\right)$ used in [2], our choice of the penalty term in (9a) is independent of $v$. So only one large tridiagonal matrix needs to be inverted later at each stage of the IMEX-RK scheme for the full discretization instead of a series of large tridiagonal matrices (the number depends on the number of discrete $v$ used in the velocity discretization). This accelerates the algorithm in practice with the same order of consistency error, already for the deterministic problems, more so for uncertain problems.

2.2.2. The IMEX implementation. Now we apply an IMEX-RK scheme to system (15), where $\left(\boldsymbol{\xi}_{1}, \boldsymbol{\eta}_{1}\right)^{T}$ is evaluated explicitly and $\left(\boldsymbol{\xi}_{2}, \boldsymbol{\eta}_{2}\right)^{T}$ implicitly; then we obtain

$$
\begin{aligned}
\hat{\mathbf{r}}^{n+1} & =\hat{\mathbf{r}}^{n}+\Delta t \sum_{k=1}^{s} \tilde{b}_{k} \boldsymbol{\xi}_{1}\left(\hat{\mathbf{R}}^{k}, \hat{\mathbf{J}}^{k}\right)+\Delta t \sum_{k=1}^{s} b_{k} \boldsymbol{\xi}_{2}\left(\hat{\mathbf{R}}^{k}\right), \\
\hat{\mathbf{j}}^{n+1} & =\hat{\mathbf{j}}^{n}+\Delta t \sum_{k=1}^{s} \tilde{b}_{k} \boldsymbol{\eta}_{1}\left(\hat{\mathbf{R}}^{k}\right)+\Delta t \sum_{k=1}^{s} b_{k} \boldsymbol{\eta}_{2}\left(\hat{\mathbf{R}}^{k}, \hat{\mathbf{J}}^{k}\right),
\end{aligned}
$$

where the internal stages are

$$
\begin{array}{r}
\hat{\mathbf{R}}^{k}=\hat{\mathbf{r}}^{n}+\Delta t \sum_{l=1}^{k-1} \tilde{a}_{k l} \boldsymbol{\xi}_{1}\left(\hat{\mathbf{R}}^{l}, \hat{\mathbf{J}}^{l}\right)+\Delta t \sum_{l=1}^{k} a_{k l} \boldsymbol{\xi}_{2}\left(\hat{\mathbf{R}}^{l}\right), \\
\hat{\mathbf{J}}^{k}=\hat{\mathbf{j}}^{n}+\Delta t \sum_{l=1}^{k-1} \tilde{a}_{k l} \boldsymbol{\eta}_{1}\left(\hat{\mathbf{R}}^{l}\right)+\Delta t \sum_{l=1}^{k} a_{k l} \boldsymbol{\eta}_{2}\left(\hat{\mathbf{R}}^{l}, \hat{\mathbf{J}}^{l}\right) .
\end{array}
$$

It is obvious that the scheme is characterized by the $s \times s$ matrices

$$
\tilde{A}=\left(\tilde{a}_{i j}\right), A=\left(a_{i j}\right)
$$

and the vectors $\tilde{b}, b \in \mathbb{R}^{s}$, which can be represented by a double table tableau in the usual Butcher notation

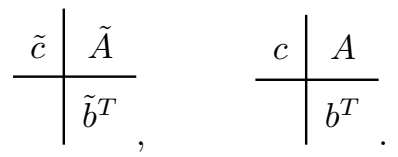

The coefficients $\tilde{c}$ and $c$ depend on the explicit part of the scheme

$$
\tilde{c}_{i}=\sum_{j=1}^{i-1} \tilde{a}_{i j}, \quad c_{i}=\sum_{j=1}^{i} a_{i j} .
$$

In the literature, there are two main different types of IMEX-RK schemes (type A and type CK) characterized by the structure of the matrix $A$. We are interested in the IMEX-RK method of type A (see [2]) where the matrix $A$ is invertible, which is not sensitive to the initial data. Of course, IMEX-RK of type CK may be used as well, where $A=\left(\begin{array}{cc}0 & 0 \\ a & \hat{A}\end{array}\right)$ with $a \in \mathbb{R}^{(s-1)}$ and the submatrix $\hat{A} \in \mathbb{R}^{(s-1) \times(s-1)}$ invertible. However, schemes of type A are more amenable to a theoretic analysis compared to type CK since the matrix $A$ of the implicit scheme is invertible. We refer to [2] for further details. 
As an example, we list the $\operatorname{SSP}(3,3,2)$ scheme, which is the second order IMEX scheme we are going to use in the numerical results

\begin{tabular}{c|cccc|ccc}
0 & 0 & 0 & 0 & $1 / 4$ & $1 / 4$ & 0 & 0 \\
$1 / 2$ & $1 / 2$ & 0 & 0 & $1 / 4$ & 0 & $1 / 4$ & 0 \\
1 & $1 / 2$ & $1 / 2$ & 0
\end{tabular}$\quad, \quad$\begin{tabular}{cccc}
$1 / 3$ & $1 / 3$ & $1 / 3$ \\
\hline & $1 / 3$ & $1 / 3$ & $1 / 3$
\end{tabular}$\quad \begin{array}{llll}1 / 3 & 1 / 3 & 1 / 3\end{array}$.

To obtain $\hat{\mathbf{R}}^{k}$ in each internal stage of (21), one needs the quantity $\hat{\mathbf{P}}^{k}=\left\langle\hat{\mathbf{R}}^{k}\right\rangle=$ $\int_{0}^{1} \hat{\mathbf{R}}^{k} d v$ in the implicit part $\boldsymbol{\xi}_{2}\left(\hat{\mathbf{R}}_{k}\right)$. This quantity can be implemented by the following procedure. Suppose one has computed $\hat{\mathbf{R}}^{l}$ for $l=1, \ldots, k-1$; then according to $(21 \mathrm{a})$

$$
\begin{aligned}
\hat{\mathbf{R}}^{k}= & \hat{\mathbf{r}}^{n}+\Delta t \sum_{l=1}^{k-1}\left[\tilde{a}_{k l} \boldsymbol{\xi}_{1}\left(\hat{\mathbf{R}}^{l}, \hat{\mathbf{J}}^{l}\right)+a_{k l} \boldsymbol{\xi}_{2}\left(\hat{\mathbf{R}}^{l}\right)\right] \\
& +\Delta t a_{k k}\left(-\frac{1}{\varepsilon^{2}} \mathbf{S}(x)\left(\hat{\mathbf{R}}^{k}-\hat{\mathbf{P}}^{k}\right)+\frac{\mu}{3} \partial_{x}\left(\mathbf{S}^{-1} \partial_{x} \hat{\mathbf{P}}^{k}\right)\right) \\
= & \hat{\mathbf{R}}^{k-1}+\Delta t a_{k k}\left(-\frac{1}{\varepsilon^{2}} \mathbf{S}(x)\left(\hat{\mathbf{R}}^{k}-\hat{\mathbf{P}}^{k}\right)+\frac{\mu}{3} \partial_{x}\left(\mathbf{S}^{-1} \partial_{x} \hat{\mathbf{P}}^{k}\right)\right) .
\end{aligned}
$$

Here $\overline{\mathbf{R}}^{k-1}$ represents all contributions in (25) from the first $k-1$ stages. Now one takes $\langle\cdot\rangle$ on both sides of $(25)$ so that $\left(\hat{\mathbf{R}}^{k}-\hat{\mathbf{P}}^{k}\right)$ is cancelled out on the right-hand side and one can obtain $\hat{\mathbf{P}}^{k}$ from the following diffusion equation in implicit form:

$$
\left.\hat{\mathbf{P}}^{k}-\Delta t a_{k k} \frac{\mu}{3} \partial_{x}\left(\mathbf{S}^{-1} \partial_{x} \hat{\mathbf{P}}^{k}\right)=\overline{\hat{\mathbf{R}}}^{k-1}\right\rangle .
$$

Then it is plugged back into (21a) in order to compute $\hat{\mathbf{R}}^{k}$.

2.3. The space discretization. To obtain the second order accuracy, we apply the upwind TVD scheme in the explicit transport part and center difference for other second derivatives. For the convenience of readers, we give the details of the spatial discretizations. During each internal stage (21),

$$
\begin{aligned}
\hat{\mathbf{R}}_{i}^{k}= & \hat{\mathbf{r}}_{i}^{n}+\Delta t \sum_{l=1}^{k-1} \tilde{a}_{k l}\left\{-\frac{v}{2 \Delta x}\left(\hat{\mathbf{J}}_{i+1}^{l}-\hat{\mathbf{J}}_{i-1}^{l}\right)+\frac{v \phi^{1 / 2}}{2 \Delta x}\left(\hat{\mathbf{R}}_{i+1}^{l}-2 \hat{\mathbf{R}}_{i}^{l}+\hat{\mathbf{R}}_{i-1}^{l}\right)\right. \\
& -\frac{v \phi^{1 / 2}}{4}\left(\gamma_{i}^{l}-\boldsymbol{\gamma}_{i-1}^{l}+\boldsymbol{\beta}_{i+1}^{l}-\boldsymbol{\beta}_{i}^{l}\right) \\
& \left.-\frac{\mu}{3 \Delta x^{2}}\left[\mathbf{S}_{i+1 / 2}^{-1}\left(\hat{\mathbf{P}}_{i+1}^{l}-\hat{\mathbf{P}}_{i}^{l}\right)-\mathbf{S}_{i-1 / 2}^{-1}\left(\hat{\mathbf{P}}_{i}^{l}-\hat{\mathbf{P}}_{i-1}^{l}\right)\right]\right\} \\
& +\Delta t \sum_{l=1}^{k} a_{k l}\left\{-\frac{1}{\varepsilon^{2}} \mathbf{S}_{i}\left(\hat{\mathbf{R}}_{i}^{l}-\hat{\mathbf{P}}_{i}^{l}\right)\right. \\
& \left.+\frac{\mu}{3 \Delta x^{2}}\left[\mathbf{S}_{i+1 / 2}^{-1}\left(\hat{\mathbf{P}}_{i+1}^{l}-\hat{\mathbf{P}}_{i}^{l}\right)-\mathbf{S}_{i-1 / 2}^{-1}\left(\hat{\mathbf{P}}_{i}^{l}-\hat{\mathbf{P}}_{i-1}^{l}\right)\right]\right\},
\end{aligned}
$$




$$
\begin{aligned}
\hat{\mathbf{J}}_{i}^{k}= & \hat{\mathbf{j}}_{i}^{n}+\Delta t \sum_{l=1}^{k-1} \tilde{a}_{k l}\left\{-\frac{v \phi}{2 \Delta x}\left(\hat{\mathbf{R}}_{i+1}^{l}-\hat{\mathbf{R}}_{i-1}^{l}\right)+\frac{v \phi^{1 / 2}}{2 \Delta x}\left(\hat{\mathbf{J}}_{i+1}^{l}-2 \hat{\mathbf{J}}_{i}^{l}+\hat{\mathbf{J}}_{i-1}^{l}\right)\right. \\
& \left.-\frac{v \phi}{4}\left(\gamma_{i}^{l}-\gamma_{i-1}^{l}-\boldsymbol{\beta}_{i+1}^{l}+\boldsymbol{\beta}_{i}^{l}\right)\right\} \\
& -\Delta t \sum_{l=1}^{k} a_{k l} \frac{1}{\varepsilon^{2}}\left\{\mathbf{S}_{i} \hat{\mathbf{J}}_{i}^{l}+\left(1-\varepsilon^{2} \phi\right) v \frac{\hat{\mathbf{R}}_{i+1}^{l}-\hat{\mathbf{R}}_{i-1}^{l}}{2 \Delta x}\right\},
\end{aligned}
$$

where

$$
\begin{aligned}
\gamma_{i}^{l}= & \frac{1}{\Delta x} \operatorname{minmod}\left(\hat{\mathbf{R}}_{i+1}^{l}+\phi^{-1 / 2} \hat{\mathbf{J}}_{i+1}^{l}-\hat{\mathbf{R}}_{i}^{l}-\phi^{-1 / 2} \hat{\mathbf{J}}_{i}^{l},\right. \\
& \left.\hat{\mathbf{R}}_{i}^{l}+\phi^{-1 / 2} \hat{\mathbf{J}}_{i}^{l}-\hat{\mathbf{R}}_{i-1}^{l}-\phi^{-1 / 2} \hat{\mathbf{J}}_{i-1}^{l}\right), \\
\boldsymbol{\beta}_{i}^{l}= & \frac{1}{\Delta x} \operatorname{minmod}\left(\hat{\mathbf{R}}_{i+1}^{l}-\phi^{-1 / 2} \hat{\mathbf{J}}_{i+1}^{l}-\hat{\mathbf{R}}_{i}^{l}+\phi^{-1 / 2} \hat{\mathbf{J}}_{i}^{l},\right. \\
& \left.\hat{\mathbf{R}}_{i}^{l}-\phi^{-1 / 2} \hat{\mathbf{J}}_{i}^{l}-\hat{\mathbf{R}}_{i-1}^{l}+\phi^{-1 / 2} \hat{\mathbf{J}}_{i-1}^{l}\right) .
\end{aligned}
$$

Since $\hat{\mathbf{P}}^{k}$ can be obtained by (26), one gets a fully discretized $\hat{\mathbf{P}}_{i}^{k}$ as follows:

$$
\hat{\mathbf{P}}_{i}^{k}-\Delta t a_{k k} \frac{\mu}{3 \Delta x^{2}}\left[\mathbf{S}_{i+1 / 2}^{-1}\left(\hat{\mathbf{P}}_{i+1}^{k}-\hat{\mathbf{P}}_{i}^{k}\right)-\mathbf{S}_{i-1 / 2}^{-1}\left(\hat{\mathbf{P}}_{i}^{k}-\hat{\mathbf{P}}_{i-1}^{k}\right)\right]=\left\langle\hat{\mathbf{R}}_{i}^{k-1}\right\rangle .
$$

By inverting a tridiagonal matrix, one can obtain $\hat{\mathbf{P}}_{i}^{k}$. Then using (29), the fully discretized $\hat{\mathbf{R}}_{i}^{k}$ is obtained and thus $\hat{\mathbf{J}}_{i}^{k}$ subsequently as

$$
\begin{aligned}
\left(\mathbf{I}+\frac{a_{k k} \Delta t}{\varepsilon^{2}} \mathbf{S}_{i}\right) \hat{\mathbf{R}}_{i}^{k}= & \hat{\mathbf{r}}_{i}^{n}+\Delta t \sum_{l=1}^{k-1} \tilde{a}_{k l}\left\{-\frac{v}{2 \Delta x}\left(\hat{\mathbf{J}}_{i+1}^{l}-\hat{\mathbf{J}}_{i-1}^{l}\right)+\frac{v \phi^{1 / 2}}{2 \Delta x}\left(\hat{\mathbf{R}}_{i+1}^{l}-2 \hat{\mathbf{R}}_{i}^{l}+\hat{\mathbf{R}}_{i-1}^{l}\right)\right. \\
& -\frac{v \phi^{1 / 2}}{4}\left(\boldsymbol{\gamma}_{i}^{l}-\boldsymbol{\gamma}_{i-1}^{l}+\boldsymbol{\beta}_{i+1}^{l}-\boldsymbol{\beta}_{i}^{l}\right) \\
& \left.-\frac{\mu}{3 \Delta x^{2}}\left[\mathbf{S}_{i+1 / 2}^{-1}\left(\hat{\mathbf{P}}_{i+1}^{l}-\hat{\mathbf{P}}_{i}^{l}\right)-\mathbf{S}_{i-1 / 2}^{-1}\left(\hat{\mathbf{P}}_{i}^{l}-\hat{\mathbf{P}}_{i-1}^{l}\right)\right]\right\} \\
& +\Delta t \sum_{l=1}^{k-1} a_{k l}\left\{-\frac{1}{\varepsilon^{2}} \mathbf{S}_{i}\left(\hat{\mathbf{R}}_{i}^{l}-\hat{\mathbf{P}}_{i}^{l}\right)\right. \\
& \left.+\frac{\mu}{3 \Delta x^{2}}\left[\mathbf{S}_{i+1 / 2}^{-1}\left(\hat{\mathbf{P}}_{i+1}^{l}-\hat{\mathbf{P}}_{i}^{l}\right)-\mathbf{S}_{i-1 / 2}^{-1}\left(\hat{\mathbf{P}}_{i}^{l}-\hat{\mathbf{P}}_{i-1}^{l}\right)\right]\right\} \\
& +\Delta t a_{k k}\left\{\frac{1}{\varepsilon^{2}} \mathbf{S}_{i} \hat{\mathbf{P}}_{i}^{k}+\frac{\mu}{3 \Delta x^{2}}\left[\mathbf{S}_{i+1 / 2}^{-1}\left(\hat{\mathbf{P}}_{i+1}^{k}-\hat{\mathbf{P}}_{i}^{k}\right)\right.\right. \\
& \left.\left.-\mathbf{S}_{i-1 / 2}^{-1}\left(\hat{\mathbf{P}}_{i}^{k}-\hat{\mathbf{P}}_{i-1}^{k}\right)\right]\right\} \\
(30 a) & \hat{\mathbf{j}}_{i}^{n}+\Delta t \sum_{l=1}^{k-1} \tilde{a}_{k l}\left\{-\frac{v \phi}{2 \Delta x}\left(\hat{\mathbf{R}}_{i+1}^{l}-\hat{\mathbf{R}}_{i-1}^{l}\right)+\frac{v \phi^{1 / 2}}{2 \Delta x}\left(\hat{\mathbf{J}}_{i+1}^{l}-2 \hat{\mathbf{J}}_{i}^{l}+\hat{\mathbf{J}}_{i-1}^{l}\right)\right. \\
\left(1+\frac{a_{k k} \Delta t}{\varepsilon^{2}} \mathbf{S}_{i}\right) \hat{\mathbf{J}}_{i}^{k}= & \left.-\frac{v \phi}{4}\left(\boldsymbol{\gamma}_{i}^{l}-\boldsymbol{\gamma}_{i-1}^{l}+\boldsymbol{\beta}_{i+1}^{l}-\boldsymbol{\beta}_{i}^{l}\right)\right\}
\end{aligned}
$$




$$
\begin{aligned}
& -\Delta t \sum_{l=1}^{k-1} a_{k l} \frac{1}{\varepsilon^{2}}\left\{\mathbf{S}_{i} \hat{\mathbf{J}}_{i}^{l}+\left(1-\varepsilon^{2} \phi\right) v \frac{\hat{\mathbf{R}}_{i+1}^{l}-\hat{\mathbf{R}}_{i-1}^{l}}{2 \Delta x}\right\} \\
& -\Delta t a_{k k}\left(\frac{1-\varepsilon^{2} \phi}{\varepsilon^{2}}\right) v \frac{\hat{\mathbf{R}}_{i+1}^{k}-\hat{\mathbf{R}}_{i-1}^{k}}{2 \Delta x} .
\end{aligned}
$$

After calculating all $\hat{\mathbf{R}}_{i}^{k}$ and $\hat{\mathbf{J}}_{i}^{k}$ for $k=1, \ldots, s$, one can update $\hat{\mathbf{r}}_{i}^{n+1}$ and $\hat{\mathbf{j}}_{i}^{n+1}$ in (20) to get

$$
\begin{aligned}
\hat{\mathbf{r}}_{i}^{n+1}= & \hat{\mathbf{r}}_{i}^{n}+\Delta t \sum_{k=1}^{s} \tilde{b}_{k}\left\{-\frac{v}{2 \Delta x}\left(\hat{\mathbf{J}}_{i+1}^{k}-\hat{\mathbf{J}}_{i-1}^{k}\right)+\frac{v \phi^{1 / 2}}{2 \Delta x}\left(\hat{\mathbf{R}}_{i+1}^{k}-2 \hat{\mathbf{R}}_{i}^{k}+\hat{\mathbf{R}}_{i-1}^{k}\right)\right. \\
& -\frac{v \phi^{1 / 2}}{4}\left(\boldsymbol{\gamma}_{i}^{k}-\boldsymbol{\gamma}_{i-1}^{k}+\boldsymbol{\beta}_{i+1}^{k}-\boldsymbol{\beta}_{i}^{k}\right) \\
& \left.-\frac{\mu}{3 \Delta x^{2}}\left[\mathbf{S}_{i+1 / 2}^{-1}\left(\hat{\mathbf{P}}_{i+1}^{k}-\hat{\mathbf{P}}_{i}^{k}\right)-\mathbf{S}_{i-1 / 2}^{-1}\left(\hat{\mathbf{P}}_{i}^{k}-\hat{\mathbf{P}}_{i-1}^{k}\right)\right]\right\} \\
& +\Delta t \sum_{k=1}^{s} b_{k}\left\{-\frac{1}{\varepsilon^{2}} \mathbf{S}_{i}\left(\hat{\mathbf{R}}_{i}^{k}-\hat{\mathbf{P}}_{i}^{k}\right)\right. \\
& \left.+\frac{\mu}{3 \Delta x^{2}}\left[\mathbf{S}_{i+1 / 2}^{-1}\left(\hat{\mathbf{P}}_{i+1}^{k}-\hat{\mathbf{P}}_{i}^{k}\right)-\mathbf{S}_{i-1 / 2}^{-1}\left(\hat{\mathbf{P}}_{i}^{k}-\hat{\mathbf{P}}_{i-1}^{k}\right)\right]\right\}, \\
\hat{\mathbf{j}}_{i}^{n+1}= & \hat{\mathbf{j}}_{i}^{n}+\Delta t \sum_{k=1}^{s} \tilde{b}_{k}\left\{-\frac{v \phi}{2 \Delta x}\left(\hat{\mathbf{R}}_{i+1}^{k}-\hat{\mathbf{R}}_{i-1}^{k}\right)+\frac{v \phi^{1 / 2}}{2 \Delta x}\left(\hat{\mathbf{J}}_{i+1}^{k}-2 \hat{\mathbf{J}}_{i}^{k}+\hat{\mathbf{J}}_{i-1}^{k}\right)\right. \\
& \left.-\frac{v \phi}{4}\left(\boldsymbol{\gamma}_{i}^{k}-\boldsymbol{\gamma}_{i-1}^{k}+\boldsymbol{\beta}_{i+1}^{k}-\boldsymbol{\beta}_{i}^{k}\right)\right\} \\
& -\Delta t \sum_{k=1}^{k} b_{k} \frac{1}{\varepsilon^{2}}\left\{\mathbf{S}_{i} \hat{\mathbf{J}}_{i}^{k}+\left(1-\varepsilon^{2} \phi\right) v \frac{\hat{\mathbf{R}}_{i+1}^{k}-\hat{\mathbf{R}}_{i-1}^{k}}{2 \Delta x}\right\},
\end{aligned}
$$

where $\boldsymbol{\gamma}_{i}^{k}$ and $\boldsymbol{\beta}_{i}^{k}$ are defined the same as in (28).

We choose [2]

$$
\mu=\exp \left(-\varepsilon^{2} / \Delta x\right)
$$

as a smoothed version of

$$
\mu(\varepsilon)=\left\{\begin{array}{lll}
1 & \text { if } & \varepsilon<\Delta x \\
0 & \text { if } & \varepsilon^{2} \geq \Delta x .
\end{array}\right.
$$

Thus, for large value of $\varepsilon$ (e.g., $\varepsilon=1$ ), one can avoid the loss of accuracy caused by adding and subtracting the penalty term; for very small value of $\varepsilon$ (e.g., $\varepsilon \rightarrow 0$ ), $\mu \rightarrow 1$.

Before continuing some observations are appropriate.

Remark 2.3.

- The velocity space is discretized by the so-called discrete ordinate method [8] which corresponds to use of the Gauss-Legendre quadrature for the velocity integrals.

- Notice that the matrix $\mathbf{S}(x)$ can be precomputed. For each stage of (20), a large tridiagonal matrix has to be inverted. Since it is sparse and can be precomputed, fast algorithms can be applied.

- To get the boundary conditions for $\hat{\mathbf{r}}$ and $\hat{\mathbf{j}}$ we refer to [20] for details. 
2.4. The sAP property. The formal definition of sAP was first defined in [21]. In this subsection, we are going to formally show that scheme (31) with internal stages (30) converges to a limiting IMEX-RK gPC-SG discretization of the diffusion equation (12) as $\varepsilon$ goes to 0 and thus scheme (31) is sAP.

Denote

$$
\begin{aligned}
\boldsymbol{\xi}_{1}\left(\hat{\mathbf{R}}_{i}^{l}, \hat{\mathbf{J}}_{i}^{l}\right)= & -\frac{v}{2 \Delta x}\left(\hat{\mathbf{J}}_{i+1}^{l}-\hat{\mathbf{J}}_{i-1}^{l}\right)+\frac{v \phi^{1 / 2}}{2 \Delta x}\left(\hat{\mathbf{R}}_{i+1}^{l}-2 \hat{\mathbf{R}}_{i}^{l}+\hat{\mathbf{R}}_{i-1}^{l}\right) \\
& -\frac{v \phi^{1 / 2}}{4}\left(\boldsymbol{\gamma}_{i}^{l}-\boldsymbol{\gamma}_{i-1}^{l}+\boldsymbol{\beta}_{i+1}^{l}-\boldsymbol{\beta}_{i}^{l}\right) \\
& -\frac{\mu}{3 \Delta x^{2}}\left[\mathbf{S}_{i+1 / 2}^{-1}\left(\hat{\mathbf{P}}_{i+1}^{l}-\hat{\mathbf{P}}_{i}^{l}\right)-\mathbf{S}_{i-1 / 2}^{-1}\left(\hat{\mathbf{P}}_{i}^{l}-\hat{\mathbf{P}}_{i-1}^{l}\right)\right], \\
\boldsymbol{\xi}_{2}\left(\hat{\mathbf{R}}_{i}^{l}\right)= & -\frac{1}{\varepsilon^{2}} \mathbf{S}_{i}\left(\hat{\mathbf{R}}_{i}^{l}-\hat{\mathbf{P}}_{i}^{l}\right) \\
& +\frac{\mu}{3 \Delta x^{2}}\left[\mathbf{S}_{i+1 / 2}^{-1}\left(\hat{\mathbf{P}}_{i+1}^{l}-\hat{\mathbf{P}}_{i}^{l}\right)-\mathbf{S}_{i-1 / 2}^{-1}\left(\hat{\mathbf{P}}_{i}^{l}-\hat{\mathbf{P}}_{i-1}^{l}\right)\right], \\
\boldsymbol{\eta}_{1}\left(\hat{\mathbf{R}}_{i}^{l}\right)= & -\frac{v \phi}{2 \Delta x}\left(\hat{\mathbf{R}}_{i+1}^{l}-\hat{\mathbf{R}}_{i-1}^{l}\right)+\frac{v \phi^{1 / 2}}{2 \Delta x}\left(\hat{\mathbf{J}}_{i+1}^{l}-2 \hat{\mathbf{J}}_{i}^{l}+\hat{\mathbf{J}}_{i-1}^{l}\right) \\
& -\frac{v \phi}{4}\left(\gamma_{i}^{l}-\boldsymbol{\gamma}_{i-1}^{l}-\boldsymbol{\beta}_{i+1}^{l}+\boldsymbol{\beta}_{i}^{l}\right), \\
\boldsymbol{\eta}_{2}\left(\hat{\mathbf{R}}_{i}^{l}, \hat{\mathbf{J}}_{i}^{l}\right)= & \frac{1}{\varepsilon^{2}}\left[\mathbf{S}_{i} \hat{\mathbf{J}}_{i}^{l}+\left(1-\varepsilon^{2} \phi\right) v \frac{\left.\hat{\mathbf{R}}_{i+1}^{l}-\hat{\mathbf{R}}_{i-1}^{l}\right]}{2 \Delta x}\right] .
\end{aligned}
$$

From (27) one gets

$$
\begin{aligned}
\left(\begin{array}{c}
\hat{\mathbf{R}}_{i}^{1} \\
\hat{\mathbf{R}}_{i}^{2} \\
\vdots \\
\hat{\mathbf{R}}_{i}^{s}
\end{array}\right) & =\left(\begin{array}{c}
\hat{\mathbf{r}}_{i}^{n} \\
\hat{\mathbf{r}}_{i}^{n} \\
\vdots \\
\vdots \\
\hat{\mathbf{r}}_{i}^{n}
\end{array}\right)+\Delta t\left(\begin{array}{c}
0 \\
\tilde{a}_{21} \boldsymbol{\xi}_{1}\left(\hat{\mathbf{R}}_{i}^{1}, \hat{\mathbf{J}}_{i}^{1}\right) \\
\vdots \\
\hat{\sum}_{l=1}^{s-1} \tilde{a}_{s l} \boldsymbol{\xi}_{1}\left(\hat{\mathbf{R}}_{i}^{l}, \hat{\mathbf{J}}_{i}^{l}\right)
\end{array}\right)+\Delta t \mathbf{A}\left(\begin{array}{c}
\boldsymbol{\xi}_{2}\left(\hat{\mathbf{R}}_{i}^{1}\right) \\
\boldsymbol{\xi}_{2}\left(\hat{\mathbf{R}}_{i}^{2}\right) \\
\vdots \\
0 \\
\boldsymbol{\xi}_{2}\left(\hat{\mathbf{R}}_{i}^{s}\right)
\end{array}\right), \\
\left(\begin{array}{c}
\hat{\mathbf{J}}_{i}^{1} \\
\hat{\mathbf{J}}_{i}^{2} \\
\vdots \\
\hat{\mathbf{J}}_{i}^{s}
\end{array}\right) & =\left(\begin{array}{c}
\hat{\mathbf{j}}_{i}^{n} \\
\hat{\mathbf{j}}_{i}^{n} \\
\vdots \\
\hat{\mathbf{j}}_{i}^{n}
\end{array}\right)+\Delta t\left(\begin{array}{c}
\tilde{\eta}_{21}\left(\hat{\mathbf{R}}_{i}, \hat{\mathbf{J}}_{i}^{1}\right) \\
\vdots \\
\left.\hat{\mathbf{R}}_{i}^{1}\right) \\
\sum_{l=1}^{s-1} \tilde{a}_{s l} \boldsymbol{\eta}_{1}\left(\hat{\mathbf{R}}_{i}^{l}\right)
\end{array}\right)+\Delta t \mathbf{A}\left(\begin{array}{c}
\boldsymbol{\eta}_{2}\left(\hat{\mathbf{R}}_{i}^{2}, \hat{\mathbf{J}}_{i}^{2}\right) \\
\vdots \\
\boldsymbol{\eta}_{2}\left(\hat{\mathbf{R}}_{i}^{s}, \hat{\mathbf{J}}_{i}^{s}\right)
\end{array}\right),
\end{aligned}
$$

where

(34) $\mathbf{A}_{K(i-1)+1: K i, K(j-1)+1: K j}=A_{i, j} \mathbf{I}_{K \times K}, \quad \mathbf{I}_{K \times K}$ is the $K \times K$ identity matrix, and $A$ is defined in (21). Denote $\mathbf{W}$ as the inverse matrix of $\mathbf{A}$, then one obtains from $(33)$

$$
\begin{gathered}
\left(\begin{array}{c}
\boldsymbol{\xi}_{2}\left(\hat{\mathbf{R}}_{i}^{1}\right) \\
\boldsymbol{\xi}_{2}\left(\hat{\mathbf{R}}_{i}^{2}\right) \\
\vdots \\
\boldsymbol{\xi}_{2}\left(\hat{\mathbf{R}}_{i}^{s}\right)
\end{array}\right)=\mathbf{W}\left[\left(\begin{array}{c}
\hat{\mathbf{R}}_{i}^{1} \\
\hat{\mathbf{R}}_{i}^{2} \\
\vdots \\
\hat{\mathbf{R}}_{i}^{s}
\end{array}\right)-\left(\begin{array}{c}
\hat{\mathbf{r}}_{i}^{n} \\
\hat{\mathbf{r}}_{i}^{n} \\
\vdots \\
\hat{\mathbf{r}}_{i}^{n}
\end{array}\right)-\Delta t\left(\begin{array}{c}
0 \\
\tilde{a}_{21} \boldsymbol{\xi}_{1}\left(\hat{\mathbf{R}}_{i}^{1}, \hat{\mathbf{J}}_{i}^{1}\right) \\
\vdots \\
\sum_{l=1}^{s-1} \tilde{a}_{s l} \boldsymbol{\xi}_{1}\left(\hat{\mathbf{R}}_{i}^{l}, \hat{\mathbf{J}}_{i}^{l}\right)
\end{array}\right)\right], \\
\Delta t\left(\begin{array}{c}
\boldsymbol{\eta}_{2}\left(\hat{\mathbf{R}}_{i}^{1}, \hat{\mathbf{J}}_{i}^{1}\right) \\
\boldsymbol{\eta}_{2}\left(\hat{\mathbf{R}}_{i}^{2}, \hat{\mathbf{J}}_{i}^{2}\right) \\
\vdots \\
\boldsymbol{\eta}_{2}\left(\hat{\mathbf{R}}_{i}^{s}, \hat{\mathbf{J}}_{i}^{s}\right)
\end{array}\right)=\mathbf{W}\left[\left(\begin{array}{c}
\hat{\mathbf{J}}_{i}^{1} \\
\hat{\mathbf{J}}_{i}^{2} \\
\vdots \\
\hat{\mathbf{J}}_{i}^{s}
\end{array}\right)-\left(\begin{array}{c}
\hat{\mathbf{j}}_{i}^{n} \\
\hat{\mathbf{j}}_{i}^{n} \\
\vdots \\
\hat{\mathbf{j}}_{i}^{n}
\end{array}\right)-\Delta t\left(\begin{array}{c}
\tilde{a}_{21} \boldsymbol{\eta}_{1}\left(\hat{\mathbf{R}}_{i}^{1}\right) \\
\vdots \\
\sum_{l=1}^{s-1} \tilde{a}_{s l} \boldsymbol{\eta}_{1}\left(\hat{\mathbf{R}}_{i}^{l}\right)
\end{array}\right)\right],
\end{gathered}
$$


Since $\mathbf{W}$ has the same structure as $\mathbf{A}, \mathbf{W}$ should be a lower triangular matrix with entries

$$
\mathbf{W}_{K(i-1)+1: K i, K(j-1)+1: K j}=\omega_{i, j} \mathbf{I}_{K \times K},
$$

where $W=\left(\omega_{i, j}\right)$ is the inverse of the lower triangular matrix $A$ in (21).

Then one can rewrite (35) as

$$
\begin{aligned}
& \Delta t \boldsymbol{\xi}_{2}\left(\hat{\mathbf{R}}_{i}^{k}\right)=\sum_{l=1}^{k} \omega_{k l}\left[\hat{\mathbf{R}}_{i}^{l}-\hat{\mathbf{r}}_{i}^{n}-\Delta t \sum_{l=1}^{k-1} \tilde{a}_{k l} \boldsymbol{\xi}_{1}\left(\hat{\mathbf{R}}_{i}^{l}, \hat{\mathbf{J}}_{i}^{l}\right)\right], \\
& \Delta t \boldsymbol{\eta}_{2}\left(\hat{\mathbf{R}}_{i}^{k}, \hat{\mathbf{J}}_{i}^{k}\right)=\sum_{l=1}^{k} \omega_{k l}\left[\hat{\mathbf{J}}_{i}^{l}-\hat{\mathbf{j}}_{i}^{n}-\Delta t \sum_{l=1}^{k-1} \tilde{a}_{k l} \boldsymbol{\eta}_{1}\left(\hat{\mathbf{R}}_{i}^{l}\right)\right] .
\end{aligned}
$$

More explicitly,

$$
\begin{aligned}
& \Delta t\left\{-\frac{1}{\varepsilon^{2}} \mathbf{S}_{i}\left(\hat{\mathbf{R}}_{i}^{k}-\hat{\mathbf{P}}_{i}^{k}\right)+\frac{\mu}{3 \Delta x^{2}}\left[\mathbf{S}_{i+1 / 2}^{-1}\left(\hat{\mathbf{P}}_{i+1}^{k}-\hat{\mathbf{P}}_{i}^{k}\right)-\mathbf{S}_{i-1 / 2}^{-1}\left(\hat{\mathbf{P}}_{i}^{k}-\hat{\mathbf{P}}_{i-1}^{k}\right)\right]\right\} \\
= & \sum_{l=1}^{k} \omega_{k l}\left\{\hat{\mathbf{R}}_{i}^{l}-\hat{\mathbf{r}}_{i}^{n}-\Delta t \sum_{l=1}^{k-1} \tilde{a}_{k l}\left[-\frac{v}{2 \Delta x}\left(\hat{\mathbf{J}}_{i+1}^{l}-\hat{\mathbf{J}}_{i-1}^{l}\right)+\frac{v \phi^{1 / 2}}{2 \Delta x}\left(\hat{\mathbf{R}}_{i+1}^{l}-2 \hat{\mathbf{R}}_{i}^{l}+\hat{\mathbf{R}}_{i-1}^{l}\right)\right.\right. \\
& \left.\left.-\frac{v \phi^{1 / 2}}{4}\left(\boldsymbol{\gamma}_{i}^{l}-\boldsymbol{\gamma}_{i-1}^{l}+\boldsymbol{\beta}_{i+1}^{l}-\boldsymbol{\beta}_{i}^{l}\right)-\frac{\mu}{3 \Delta x^{2}}\left(\mathbf{S}_{i+1 / 2}^{-1}\left(\hat{\mathbf{P}}_{i+1}^{l}-\hat{\mathbf{P}}_{i}^{l}\right)-\mathbf{S}_{i-1 / 2}^{-1}\left(\hat{\mathbf{P}}_{i}^{l}-\hat{\mathbf{P}}_{i-1}^{l}\right)\right)\right]\right\},
\end{aligned}
$$

$$
\begin{aligned}
& \Delta t\left\{\frac{1}{\varepsilon^{2}}\left[\mathbf{S}_{i} \hat{\mathbf{J}}_{i}^{k}+\left(1-\varepsilon^{2} \phi\right) v \frac{\hat{\mathbf{R}}_{i+1}^{k}-\hat{\mathbf{R}}_{i-1}^{k}}{2 \Delta x}\right]\right\} \\
= & \sum_{l=1}^{k} \omega_{k l}\left\{\hat{\mathbf{J}}_{i}^{l}-\hat{\mathbf{j}}_{i}^{n}-\Delta t \sum_{l=1}^{k-1} \tilde{a}_{k l}\left[-\frac{v \phi}{2 \Delta x}\left(\hat{\mathbf{R}}_{i+1}^{l}-\hat{\mathbf{R}}_{i-1}^{l}\right)+\frac{v \phi^{1 / 2}}{2 \Delta x}\left(\hat{\mathbf{J}}_{i+1}^{l}-2 \hat{\mathbf{J}}_{i}^{l}+\hat{\mathbf{J}}_{i-1}^{l}\right)\right.\right. \\
& \left.\left.-\frac{v \phi}{4}\left(\boldsymbol{\gamma}_{i}^{l}-\gamma_{i-1}^{l}-\boldsymbol{\beta}_{i+1}^{l}+\boldsymbol{\beta}_{i}^{l}\right)\right]\right\} .
\end{aligned}
$$

Thus, setting $\varepsilon \rightarrow 0$, since $\mathbf{S}_{i}$ is nonsingular, one obtains

$$
\begin{aligned}
\hat{\mathbf{R}}_{i}^{k} & =\hat{\mathbf{P}}_{i}^{k}, \\
\hat{\mathbf{J}}_{i}^{k} & =-v \mathbf{S}_{i}^{-1} \frac{\hat{\mathbf{R}}_{i+1}^{k}-\hat{\mathbf{R}}_{i-1}^{k}}{2 \Delta x} .
\end{aligned}
$$

Inserting this back into (31a) and letting $\varepsilon \rightarrow 0$,

$$
\hat{\mathbf{r}}_{i}^{n+1}=\hat{\mathbf{r}}_{i}^{n}+\Delta t \sum_{k=1}^{s} \tilde{b}_{k} \hat{\boldsymbol{\xi}}_{1}\left(\hat{\mathbf{R}}_{i}^{k}\right)+\Delta t \sum_{k=1}^{s} b_{k} \hat{\boldsymbol{\xi}}_{2}\left(\hat{\mathbf{R}}_{i}^{k}\right),
$$

where

$$
\begin{aligned}
\hat{\boldsymbol{\xi}_{1}}\left(\hat{\mathbf{R}}_{i}^{k}\right)= & v^{2} \frac{1}{4 \Delta x^{2}}\left[\mathbf{S}_{i+1}^{-1}\left(\hat{\mathbf{R}}_{i+2}^{k}-\hat{\mathbf{R}}_{i}^{k}\right)-\mathbf{S}_{i-1}^{-1}\left(\hat{\mathbf{R}}_{i}^{k}-\hat{\mathbf{R}}_{i-2}^{k}\right)\right] \\
& -\frac{1}{3 \Delta x^{2}}\left[\mathbf{S}_{i+1 / 2}^{-1}\left(\hat{\mathbf{R}}_{i+1}^{k}-\hat{\mathbf{R}}_{i}^{k}\right)-\mathbf{S}_{i-1 / 2}^{-1}\left(\hat{\mathbf{R}}_{i}^{k}-\hat{\mathbf{R}}_{i-1}^{k}\right)\right], \\
\hat{\boldsymbol{\xi}}_{2}\left(\hat{\mathbf{R}}_{i}^{k}\right)= & \frac{1}{3 \Delta x^{2}}\left[\mathbf{S}_{i+1 / 2}^{-1}\left(\hat{\mathbf{R}}_{i+1}^{k}-\hat{\mathbf{R}}_{i}^{k}\right)-\mathbf{S}_{i-1 / 2}^{-1}\left(\hat{\mathbf{R}}_{i}^{k}-\hat{\mathbf{R}}_{i-1}^{k}\right)\right] .
\end{aligned}
$$


Integrating above over $v$, one gets

$$
\hat{\boldsymbol{\rho}}_{i}^{n+1}=\hat{\boldsymbol{\rho}}_{i}^{n}+\frac{\Delta t}{3 \Delta x^{2}} \sum_{k=1}^{s} b_{k}\left[\mathbf{S}_{i+1 / 2}^{-1}\left(\hat{\mathbf{P}}_{i+1}^{k}-\hat{\mathbf{P}}_{i}^{k}\right)-\mathbf{S}_{i-1 / 2}^{-1}\left(\hat{\mathbf{P}}_{i}^{k}-\hat{\mathbf{P}}_{i-1}^{k}\right)\right]+O\left(\Delta x^{2}\right)
$$

where

$$
\hat{\mathbf{P}}_{i}^{k}=\hat{\boldsymbol{\rho}}_{i}^{n}+\frac{\Delta t}{3 \Delta x^{2}} \sum_{l=1}^{k} a_{k l}\left[\mathbf{S}_{i+1 / 2}^{-1}\left(\hat{\mathbf{P}}_{i+1}^{l}-\hat{\mathbf{P}}_{i}^{l}\right)-\mathbf{S}_{i-1 / 2}^{-1}\left(\hat{\mathbf{P}}_{i}^{l}-\hat{\mathbf{P}}_{i-1}^{l}\right)\right]
$$

which is an implicit RK scheme (requiring inverting a tridiagonal matrix at each stage) for the limiting IMEX-RK gPC-SG approximation of the diffusion equation (12) and also corresponds to (29). Thus, the sAP property [21] of the efficient IMEX R-K scheme is shown.

3. The radiative heat transfer equation with random inputs. Let $x \in$ $D \subset \mathbb{R}^{3}$ be the space variable, $\Omega \in S^{2}$ be the direction variable, $S^{2}$ be the unit sphere of $\mathbb{R}^{3}, z \in \mathbb{R}^{d}(d \geq 1)$ be the random variable, and $t \in \mathbb{R}^{+}$be the time.

We denote by $I=I(x, \Omega, z, t)$ the radiative intensity and by $\theta(x, z, t)$ the material temperature. Introducing the Knudsen number $\varepsilon$, the radiative heat transfer equations in nondimensional form are

$$
\begin{aligned}
& \varepsilon^{2} M \partial_{t} I+\varepsilon \Omega \cdot \nabla_{x} I=B(\theta)-I, \\
& \varepsilon^{2} \partial_{t} \theta=\varepsilon^{2} \Delta_{x} \theta-(B(\theta)-\langle I\rangle)
\end{aligned}
$$

with the total intensity

$$
\langle I\rangle(x, z, t)=\frac{1}{|S|^{2}} \int_{S^{2}} I(x, \Omega, z, t) d \Omega
$$

and the black body intensity

$$
B(\theta)=\sigma \theta^{4},
$$

where $M$ is the Mach number (= 1 for this paper) and $\sigma=\sigma(x, z)>0$ is the cross section depending on the space variable and the random variable.

The initial conditions and reflection transmission boundary conditions are prescribed as follows:

$$
\begin{aligned}
& \text { I.C. }\left\{\begin{array}{l}
I(x, \Omega, z, 0)=I_{I}(x, \Omega, z), \\
\theta(x, z, 0)=\theta_{I}(x, z),
\end{array}\right. \\
& \text { B.C. }\left\{\begin{array}{l}
I(\hat{x}, \Omega, z, t)=\alpha(n(\hat{x}) \cdot \Omega) I\left(\hat{x}, \Omega^{\prime}, z, t\right)+[1-\alpha(n(\hat{x}) \cdot \Omega)] I_{B}(\hat{x}, \Omega, z, t), \\
\text { for } n(\hat{x}) \cdot \Omega<0, \\
\theta(\hat{x}, z, t)=\theta_{B}(\hat{x}, z, t),
\end{array}\right.
\end{aligned}
$$

where $\hat{x} \in \partial D$ with outward unit normal $n(\hat{x})$ and $\Omega^{\prime}=\Omega-2 n(\hat{x})(n(\hat{x}) \cdot \Omega)$ is the reflection of $\Omega$ on the tangent plane to $\partial D$. The reflectivity $\alpha, 0 \leq \alpha \leq 1$, depends on the incidence angle. These boundary conditions avoid the formation of a boundary layer for $\varepsilon \ll 1$ [23], which is numerically difficult for AP schemes [16].

3.1. An even-odd decomposition. For simplicity, we consider the onedimensional case $x \in[0,1]$ and define $v=\cos (\Omega \cdot x), v \in[-1,1]$. Thus, the angular averaging is defined as 


$$
\langle f\rangle=\frac{1}{2} \int_{-1}^{1} f(v) d v .
$$

The one-dimensional radiative heat transfer equation is

$$
\begin{aligned}
& \varepsilon^{2} \partial_{t} I+\varepsilon v \partial_{x} I=B(\theta)-I, \\
& \varepsilon^{2} \partial_{t} \theta=\varepsilon^{2} \partial_{x x} \theta-(B(\theta)-\langle I\rangle) .
\end{aligned}
$$

For $v>0$,

$$
\begin{aligned}
& \varepsilon^{2} \partial_{t} I(v)+\varepsilon v \partial_{x} I(v)=B(\theta)-I(v), \\
& \varepsilon^{2} \partial_{t} I(-v)-\varepsilon v \partial_{x} I(-v)=B(\theta)-I(-v), \\
& \varepsilon^{2} \partial_{t} \theta=\varepsilon^{2} \partial_{x x} \theta-(B(\theta)-\langle I\rangle) .
\end{aligned}
$$

Now denote the even and odd parities

$$
\begin{aligned}
r(t, x, v, z) & =\frac{1}{2}(I(t, x, v, z)+I(t, x,-v, z)), \\
j(t, x, v, z) & =\frac{1}{2 \varepsilon}(I(t, x, v, z)-I(t, x,-v, z)) .
\end{aligned}
$$

Then (49) becomes

$$
\begin{aligned}
& \partial_{t} r+v \partial_{x} j=\frac{1}{\varepsilon^{2}}(B(\theta)-r), \\
& \partial_{t} j+\frac{1}{\varepsilon^{2}} v \partial_{x} r=-\frac{1}{\varepsilon^{2}} j, \\
& \partial_{t} \theta=\partial_{x x} \theta-\frac{1}{\varepsilon^{2}}(B(\theta)-\langle r\rangle) .
\end{aligned}
$$

As $\varepsilon \rightarrow 0,(51)$ gives

$$
B(\theta)=r=\langle r\rangle, \quad j=-v \partial_{x} r .
$$

Applying this in (51a), integrating over $v$, and adding to (51c)

$$
\partial_{t}(\theta+B(\theta))=\partial_{x}\left[\partial_{x} \theta+\frac{1}{3} \partial_{x} B(\theta)\right],
$$

which is the same limiting diffusion equation one can derive from the Hilbert expansion as $[23]$.

3.2. The gPC-SG formulation. Now we deal with the uncertainty. Using the gPC approximation for $r(t, x, v, z), j(t, x, v, z)$, and $\theta(t, x, z)$ and truncating at the $N$ th order,

$$
\begin{aligned}
& r(t, x, v, z) \approx r_{N}(t, x, v, z)=\sum_{k=1}^{K} \hat{r}_{k}(t, x, v) \Phi_{k}(z)=\hat{\mathbf{r}} \cdot \boldsymbol{\Phi} \\
& j(t, x, v, z) \approx j_{N}(t, x, v, z)=\sum_{k=1}^{K} \hat{j}_{k}(t, x, v) \Phi_{k}(z)=\hat{\mathbf{j}} \cdot \boldsymbol{\Phi} \\
& \theta(t, x, z) \approx \theta_{N}(t, x, z)=\sum_{k=1}^{K} \hat{\theta}_{k}(t, x) \Phi_{k}(z)=\hat{\boldsymbol{\theta}} \cdot \mathbf{\Phi}
\end{aligned}
$$


where $\Phi_{k}$ is the same as defined in (8) and

$$
\begin{aligned}
& \hat{\boldsymbol{r}}=\left(\hat{r}_{1}, \hat{r}_{2}, \ldots, \hat{r}_{K}\right)^{T}, \\
& \hat{\boldsymbol{j}}=\left(\hat{j}_{1}, \hat{j}_{2}, \ldots, \hat{j}_{K}\right)^{T}, \\
& \hat{\boldsymbol{\theta}}=\left(\hat{\theta}_{1}, \hat{\theta}_{2}, \ldots, \hat{\theta}_{K}\right)^{T}, \\
& \hat{\boldsymbol{\Phi}}=\left(\hat{\Phi}_{1}, \hat{\Phi}_{2}, \ldots, \hat{\Phi}_{K}\right)^{T} .
\end{aligned}
$$

Then (51) becomes

$$
\begin{aligned}
& \partial_{t} \hat{\mathbf{r}}+v \partial_{x} \hat{\mathbf{j}}=\frac{1}{\varepsilon^{2}}(\mathbf{B}(\hat{\boldsymbol{\theta}})-\hat{\mathbf{r}}), \\
& \partial_{t} \hat{\mathbf{j}}+\frac{1}{\varepsilon^{2}} v \partial_{x} \hat{\mathbf{r}}=-\frac{1}{\varepsilon^{2}} \hat{\mathbf{j}}, \\
& \partial_{t} \hat{\boldsymbol{\theta}}=\partial_{x x} \hat{\boldsymbol{\theta}}-\frac{1}{\varepsilon^{2}}(\mathbf{B}(\hat{\boldsymbol{\theta}})-\langle\hat{\mathbf{r}}\rangle),
\end{aligned}
$$

where $\mathbf{B}(\hat{\boldsymbol{\theta}})=\left(B_{j}\right)_{1 \leq j \leq K}$ with

$$
B_{j}=\int_{I_{z}}(\hat{\boldsymbol{\theta}} \cdot \mathbf{\Phi})^{4} \sigma(x, z) \Phi_{j}(z) \pi(z) d z .
$$

Similarly, the limiting diffusion equation (53) becomes

$$
\partial_{t}[\hat{\boldsymbol{\theta}}+\mathbf{B}(\hat{\boldsymbol{\theta}})]=\partial_{x}\left[\left(\mathbf{I}+\frac{4}{3} \mathbf{C}\right) \partial_{x} \hat{\boldsymbol{\theta}}\right],
$$

where $\mathbf{I}$ is a $K \times K$ identity matrix and $\mathbf{C}(\hat{\boldsymbol{\theta}})=\left(c_{i j}\right)_{1 \leq i, j \leq K}$ with

$$
c_{i j}=\int_{I_{z}}(\hat{\boldsymbol{\theta}} \cdot \boldsymbol{\Phi})^{3} \sigma(x, z) \Phi_{i}(z) \Phi_{j}(z) \pi(z) d z .
$$

The well-posedness of this equation under some gentle condition is proved in [19].

3.3. An efficient IMEX-RK scheme. One could apply the same relaxation method as in [20] for system (56), but it will still suffer from the parabolic CFL condition $\Delta t=O\left(\Delta x^{2}\right)$. So similarly as in section 2.2, we rewrite (56) as follows:

$$
\begin{aligned}
& \partial_{t} \hat{\mathbf{r}}=-v \partial_{x} \hat{\mathbf{j}}+\frac{1}{\varepsilon^{2}}(\mathbf{B}(\hat{\boldsymbol{\theta}})-\hat{\mathbf{r}}), \\
& \partial_{t} \hat{\mathbf{j}}=-\frac{1}{\varepsilon^{2}}\left(\hat{\mathbf{j}}+v \partial_{x} \hat{\mathbf{r}}\right), \\
& \partial_{t} \hat{\boldsymbol{\theta}}=\partial_{x x} \hat{\boldsymbol{\theta}}-\frac{1}{\varepsilon^{2}}(\mathbf{B}(\hat{\boldsymbol{\theta}})-\langle\hat{\mathbf{r}}\rangle) .
\end{aligned}
$$

By adding and subtracting the term $\frac{\mu}{3} \partial_{x x} \mathbf{B}(\hat{\boldsymbol{\theta}})$ in (60a) and the term $\phi v \partial_{x} \hat{\mathbf{r}}$ in (60b), we reformulate the problem into its equivalent form

$$
\begin{aligned}
\partial_{t} \hat{\mathbf{r}} & =\left[-v \partial_{x} \hat{\mathbf{j}}-\frac{\mu}{3} \partial_{x x} \mathbf{B}(\hat{\boldsymbol{\theta}})\right]+\left[\frac{1}{\varepsilon^{2}}(\mathbf{B}(\hat{\boldsymbol{\theta}})-\hat{\mathbf{r}})+\frac{\mu}{3} \partial_{x x} \mathbf{B}(\hat{\boldsymbol{\theta}})\right], \\
& =\boldsymbol{\xi}_{1}(\hat{\mathbf{j}}, \hat{\boldsymbol{\theta}})+\boldsymbol{\xi}_{2}(\hat{\mathbf{r}}, \hat{\boldsymbol{\theta}}), \\
\partial_{t} \hat{\mathbf{j}} & \left.=-v \phi \partial_{x} \hat{\mathbf{r}}-\frac{1}{\varepsilon^{2}} \hat{\mathbf{j}}+\left(1-\varepsilon^{2} \phi\right) v \partial_{x} \hat{\mathbf{r}}\right]=\boldsymbol{\eta}_{1}(\hat{\mathbf{r}})+\boldsymbol{\eta}_{2}(\hat{\mathbf{r}}, \hat{\mathbf{j}}), \\
\partial_{t} \hat{\boldsymbol{\theta}} & =\partial_{x x} \hat{\boldsymbol{\theta}}-\frac{1}{\varepsilon^{2}}(\mathbf{B}(\hat{\boldsymbol{\theta}})-\langle\hat{\mathbf{r}}\rangle)=h(\hat{\boldsymbol{\theta}}),
\end{aligned}
$$


where

$$
\begin{aligned}
& \boldsymbol{\xi}_{1}(\hat{\mathbf{j}}, \hat{\boldsymbol{\theta}})=-v \partial_{x} \hat{\mathbf{j}}-\frac{\mu}{3} \partial_{x x} \mathbf{B}(\hat{\boldsymbol{\theta}}), \\
& \boldsymbol{\xi}_{2}(\hat{\mathbf{r}}, \hat{\boldsymbol{\theta}})=\frac{1}{\varepsilon^{2}}(\mathbf{B}(\hat{\boldsymbol{\theta}})-\hat{\mathbf{r}})+\frac{\mu}{3} \partial_{x x} \mathbf{B}(\hat{\boldsymbol{\theta}}), \\
& \boldsymbol{\eta}_{1}(\hat{\mathbf{r}})=-v \phi \partial_{x} \hat{\mathbf{r}} \\
& \boldsymbol{\eta}_{2}(\hat{\mathbf{r}}, \hat{\mathbf{j}})=-\frac{1}{\varepsilon^{2}}\left[\hat{\mathbf{j}}+\left(1-\varepsilon^{2} \phi\right) v \partial_{x} \hat{\mathbf{r}}\right] .
\end{aligned}
$$

The constants $\mu$ and $\phi$ are chosen the same as in (17) and (19), respectively.

3.3.1. The IMEX-RK method. Now we apply an IMEX-RK scheme to system (61) where $\left(\boldsymbol{\xi}_{1}, \boldsymbol{\eta}_{1}, 0\right)^{T}$ is evaluated explicitly and $\left(\boldsymbol{\xi}_{2}, \boldsymbol{\eta}_{2}, h\right)^{T}$ implicitly; then we obtain

$$
\begin{aligned}
\hat{\mathbf{r}}^{n+1} & =\hat{\mathbf{r}}^{n}+\Delta t \sum_{k=1}^{s} \tilde{b}_{k} \boldsymbol{\xi}_{1}\left(\hat{\mathbf{J}}^{k}, \hat{\boldsymbol{\Theta}}^{k}\right)+\Delta t \sum_{k=1}^{s} b_{k} \boldsymbol{\xi}_{2}\left(\hat{\mathbf{R}}^{k}, \hat{\boldsymbol{\Theta}}^{k}\right), \\
\hat{\mathbf{j}}^{n+1} & =\hat{\mathbf{j}}^{n}+\Delta t \sum_{k=1}^{s} \tilde{b}_{k} \boldsymbol{\eta}_{1}\left(\hat{\mathbf{R}}^{k}\right)+\Delta t \sum_{k=1}^{s} b_{k} \boldsymbol{\eta}_{2}\left(\hat{\mathbf{R}}^{k}, \hat{\mathbf{J}}^{k}\right), \\
\hat{\boldsymbol{\theta}}^{n+1} & =\hat{\boldsymbol{\theta}}^{n}+\Delta t \sum_{k=1}^{s} b_{k} h\left(\hat{\boldsymbol{\Theta}}^{k}\right),
\end{aligned}
$$

where the internal stages are

$$
\begin{aligned}
& \hat{\mathbf{R}}^{k}=\hat{\mathbf{r}}^{n}+\Delta t \sum_{l=1}^{k-1} \tilde{a}_{k l} \boldsymbol{\xi}_{1}\left(\hat{\mathbf{J}}^{l}, \hat{\boldsymbol{\Theta}}^{l}\right)+\Delta t \sum_{l=1}^{k} a_{k l} \boldsymbol{\xi}_{2}\left(\hat{\boldsymbol{R}}^{l}, \hat{\boldsymbol{\Theta}}^{l}\right), \\
& \hat{\mathbf{J}}^{k}=\hat{\mathbf{j}}^{n}+\Delta t \sum_{l=1}^{k-1} \tilde{a}_{k l} \boldsymbol{\eta}_{1}\left(\hat{\mathbf{R}}^{l}\right)+\Delta t \sum_{l=1}^{k} a_{k l} \boldsymbol{\eta}_{2}\left(\hat{\mathbf{R}}^{l}, \hat{\mathbf{J}}^{l}\right), \\
& \hat{\boldsymbol{\Theta}}^{k}=\hat{\boldsymbol{\theta}}^{n}+\Delta t \sum_{l=1}^{k} a_{k l} h\left(\hat{\boldsymbol{\Theta}}^{l}\right) .
\end{aligned}
$$

Notice that for each internal stage, one has to compute $\hat{\boldsymbol{\Theta}}_{k}$ first. Then $\hat{\mathbf{R}}_{k}$ and $\hat{\mathbf{J}}_{k}$ can be solved similarly as before:

$$
\hat{\boldsymbol{\Theta}}^{k}=\hat{\boldsymbol{\theta}}^{n}+\Delta t \sum_{l=1}^{k} a_{k l}\left(\partial_{x x} \hat{\boldsymbol{\Theta}}^{l}-\frac{1}{\varepsilon^{2}}\left(\mathbf{B}\left(\hat{\boldsymbol{\Theta}}^{l}\right)-\left\langle\hat{\boldsymbol{R}}^{l}\right\rangle\right)\right) .
$$

Take $\langle\cdot\rangle$ on $(64 a)$,

$$
\begin{aligned}
\left\langle\hat{\mathbf{R}}^{k}\right\rangle= & \left\langle\hat{\mathbf{r}}^{n}+\Delta t \sum_{l=1}^{k-1} \tilde{a}_{k l} \boldsymbol{\xi}_{1}\left(\hat{\mathbf{J}}^{l}, \hat{\boldsymbol{\Theta}}^{l}\right)+\Delta t \sum_{l=1}^{k-1} a_{k l} \boldsymbol{\xi}_{2}\left(\hat{\mathbf{R}}^{l}, \hat{\boldsymbol{\Theta}}^{l}\right)\right\rangle+\Delta t a_{k k}\left\langle\boldsymbol{\xi}_{2}\left(\hat{\mathbf{R}}^{k}, \hat{\boldsymbol{\Theta}}^{k}\right)\right\rangle \\
= & \left\langle\hat{\mathbf{r}}^{n}+\Delta t \sum_{l=1}^{k-1} \tilde{a}_{k l} \boldsymbol{\xi}_{1}\left(\hat{\mathbf{J}}^{l}, \hat{\boldsymbol{\Theta}}^{l}\right)+\Delta t \sum_{l=1}^{k-1} a_{k l} \boldsymbol{\xi}_{2}\left(\hat{\mathbf{R}}^{l}, \hat{\boldsymbol{\Theta}}^{l}\right)\right\rangle \\
& +\Delta t a_{k k}\left(\frac{1}{\varepsilon^{2}}\left(\mathbf{B}\left(\hat{\boldsymbol{\Theta}}^{k}\right)-\left\langle\hat{\mathbf{R}}^{k}\right\rangle\right)+\frac{\mu}{3} \partial_{x x} \mathbf{B}\left(\hat{\boldsymbol{\Theta}}^{k}\right)\right) .
\end{aligned}
$$


Add (65) and (66),

$$
\begin{aligned}
\hat{\boldsymbol{\Theta}}^{k}+\left\langle\hat{\mathbf{R}}^{k}\right\rangle= & \hat{\boldsymbol{\Theta}}^{n}+\Delta t \sum_{l=1}^{k-1} a_{k l} h\left(\hat{\boldsymbol{\Theta}}^{l}\right) \\
& +\left\langle\hat{\mathbf{r}}^{n}+\Delta t \sum_{l=1}^{k-1} \tilde{a}_{k l} \boldsymbol{\xi}_{1}\left(\hat{\mathbf{J}}^{l}, \hat{\boldsymbol{\Theta}}^{l}\right)+\Delta t \sum_{l=1}^{k-1} a_{k l} \boldsymbol{\xi}_{2}\left(\hat{\mathbf{R}}^{l}, \hat{\boldsymbol{\Theta}}^{l}\right)\right\rangle \\
& +\Delta t a_{k k}\left(\partial_{x x} \hat{\boldsymbol{\Theta}}^{k}+\frac{\mu}{3} \partial_{x x} \mathbf{B}\left(\hat{\boldsymbol{\Theta}}^{k}\right)\right) .
\end{aligned}
$$

Thus, $\left\langle\hat{\mathbf{R}}^{k}\right\rangle$ can be expressed in terms of $\hat{\boldsymbol{\Theta}}^{k}$ and other explicit terms. Inserting it back into (65), then

(68)

$$
\begin{aligned}
\hat{\boldsymbol{\Theta}}^{k}= & \hat{\boldsymbol{\Theta}}^{n}+\Delta t \sum_{l=1}^{k-1} a_{k l} h\left(\hat{\boldsymbol{\Theta}}^{l}\right)+\Delta t a_{k k} \partial_{x x} \hat{\boldsymbol{\Theta}}^{k}-\frac{\Delta t a_{k k}}{\varepsilon^{2}} \mathbf{B}\left(\hat{\boldsymbol{\Theta}}^{k}\right) \\
& +\frac{\Delta t a_{k k}}{\varepsilon^{2}}\left[\hat{\boldsymbol{\Theta}}^{n}+\Delta t \sum_{l=1}^{k-1} a_{k l} h\left(\hat{\boldsymbol{\Theta}}^{l}\right)+\left\langle\hat{\mathbf{r}}^{n}+\Delta t \sum_{l=1}^{k-1} \tilde{a}_{k l} \boldsymbol{\xi}_{1}\left(\hat{\mathbf{J}}^{l}, \hat{\boldsymbol{\Theta}}^{l}\right)+\Delta t \sum_{l=1}^{k-1} a_{k l} \boldsymbol{\xi}_{2}\left(\hat{\mathbf{R}}^{l}, \hat{\boldsymbol{\Theta}}^{l}\right)\right\rangle\right. \\
& \left.+\Delta t a_{k k}\left(\partial_{x x} \hat{\boldsymbol{\Theta}}^{k}+\frac{\mu}{3} \partial_{x x} \mathbf{B}\left(\hat{\boldsymbol{\Theta}}^{k}\right)\right)-\hat{\boldsymbol{\Theta}}^{k}\right] .
\end{aligned}
$$

Reordering and approximating $\mathbf{B}\left(\hat{\boldsymbol{\Theta}}^{k}\right)$ by $\mathbf{B}\left(\hat{\boldsymbol{\Theta}}^{k-1}\right)+4 \mathbf{C}\left(\hat{\boldsymbol{\Theta}}^{k-1}\right)\left(\hat{\boldsymbol{\Theta}}^{k}-\hat{\boldsymbol{\Theta}}^{k-1}\right)$ and $\partial_{x x} \mathbf{B}\left(\hat{\boldsymbol{\Theta}}^{k}\right)$ by $\partial_{x}\left(4 \mathbf{C}\left(\hat{\boldsymbol{\Theta}}^{k-1}\right) \partial_{x} \hat{\boldsymbol{\Theta}}^{k}\right)$, one gets

$$
\begin{aligned}
& {\left[\left(1+\frac{\Delta t a_{k k}}{\varepsilon^{2}}\right) \mathbf{I}+\frac{\Delta t a_{k k}}{\varepsilon^{2}} 4 \mathbf{C}\left(\hat{\boldsymbol{\Theta}}^{k-1}\right)\right] \hat{\boldsymbol{\Theta}}^{k}-\Delta t a_{k k}\left(1+\frac{\Delta t a_{k k}}{\varepsilon^{2}}\right) \partial_{x x} \hat{\boldsymbol{\Theta}}^{k} } \\
& -\frac{\left(\Delta t a_{k k}\right)^{2}}{\varepsilon^{2}} \frac{4 \mu}{3} \partial_{x}\left(\mathbf{C}\left(\hat{\boldsymbol{\Theta}}^{k-1}\right) \partial_{x} \hat{\boldsymbol{\Theta}}^{k}\right) \\
= & \left(1+\frac{\Delta t a_{k k}}{\varepsilon^{2}}\right) \hat{\boldsymbol{\Theta}}^{n}+\left(1+\frac{\Delta t a_{k k}}{\varepsilon^{2}}\right) \Delta t \sum_{l=1}^{k-1} a_{k l} h\left(\hat{\boldsymbol{\Theta}}^{l}\right) \\
& +\frac{\Delta t a_{k k}}{\varepsilon^{2}}\left[\left\langle\hat{\mathbf{r}}^{n}+\Delta t \sum_{l=1}^{k-1} \tilde{a}_{k l} \boldsymbol{\xi}_{1}\left(\hat{\mathbf{J}}^{l}, \hat{\boldsymbol{\Theta}}^{l}\right)+\Delta t \sum_{l=1}^{k-1} a_{k l} \boldsymbol{\xi}_{2}\left(\hat{\mathbf{R}}^{l}, \hat{\boldsymbol{\Theta}}^{l}\right)\right\rangle+3 \mathbf{B}\left(\hat{\boldsymbol{\Theta}}^{k-1}\right)\right],
\end{aligned}
$$

where $\mathbf{I}$ is a $K \times K$ identity matrix, and $\mathbf{B}$ and $\mathbf{C}$ are the same as (57) and (59).

3.3.2. The spatial discretization. Following the same space discretization as in section 2.3 ,

$$
\begin{aligned}
& {\left[\left(1+\frac{\Delta t a_{k k}}{\varepsilon^{2}}\right) \mathbf{I}+\frac{\Delta t a_{k k}}{\varepsilon^{2}} 4 \mathbf{C}(\hat{\boldsymbol{\Theta}})_{i}^{k}\right] \hat{\boldsymbol{\Theta}}_{i}^{k}-\Delta t a_{k k}\left(1+\frac{\Delta t a_{k k}}{\varepsilon^{2}}\right) \frac{\hat{\boldsymbol{\Theta}}_{i+1}^{k}-2 \hat{\boldsymbol{\Theta}}_{i}^{k}+\hat{\boldsymbol{\Theta}}_{i-1}^{k}}{\Delta x^{2}}} \\
& -\frac{\left(\Delta t a_{k k}\right)^{2}}{\varepsilon^{2}} \frac{4 \mu}{3} \frac{\mathbf{C}(\hat{\boldsymbol{\Theta}})_{i+1 / 2}^{k-1}\left(\hat{\boldsymbol{\Theta}}_{i+1}^{k}-\hat{\boldsymbol{\Theta}}_{i}^{k}\right)-\mathbf{C}(\hat{\boldsymbol{\Theta}})_{i-1 / 2}^{k-1}\left(\hat{\boldsymbol{\Theta}}_{i}^{k}-\hat{\boldsymbol{\Theta}}_{i-1}^{k}\right)}{\Delta x^{2}}
\end{aligned}
$$




$$
\begin{aligned}
= & \left(1+\frac{\Delta t a_{k k}}{\varepsilon^{2}}\right) \hat{\boldsymbol{\theta}}_{i}^{n}+\left(1+\frac{\Delta t a_{k k}}{\varepsilon^{2}}\right) \Delta t \sum_{l=1}^{k-1} a_{k l} h\left(\hat{\mathbf{\Theta}}_{i}^{l}\right) \\
& +\frac{\Delta t a_{k k}}{\varepsilon^{2}}\left[\left\langle\hat{\mathbf{r}}_{i}^{n}+\Delta t \sum_{l=1}^{k-1} \tilde{a}_{k l} \boldsymbol{\xi}_{1}\left(\hat{\mathbf{J}}_{i}^{l}, \hat{\mathbf{\Theta}}_{i}^{l}\right)+\Delta t \sum_{l=1}^{k-1} a_{k l} \boldsymbol{\xi}_{2}\left(\hat{\mathbf{R}}_{i}^{l}, \hat{\mathbf{\Theta}}_{i}^{l}\right)\right\rangle+3 \mathbf{B}\left(\hat{\mathbf{\Theta}}_{i}^{k-1}\right)\right],
\end{aligned}
$$

where

$$
\begin{aligned}
& h\left(\hat{\boldsymbol{\Theta}}_{i}^{l}\right)=\frac{\hat{\boldsymbol{\Theta}}_{i+1}^{l}-2 \hat{\boldsymbol{\Theta}}_{i}^{l}+\hat{\boldsymbol{\Theta}}_{i-1}^{l}}{\Delta x^{2}}-\frac{1}{\varepsilon^{2}}\left(\mathbf{B}\left(\hat{\boldsymbol{\Theta}}_{i}^{l}\right)-\left\langle\hat{\mathbf{R}}_{i}^{l}\right\rangle\right), \\
& \boldsymbol{\xi}_{1}\left(\hat{\mathbf{J}}_{i}^{l}, \hat{\boldsymbol{\Theta}}_{i}^{l}\right)=-\frac{v}{2 \Delta x}\left(\hat{\mathbf{J}}_{i+1}^{l}-\hat{\mathbf{J}}_{i-1}^{l}\right)+\frac{v \phi^{1 / 2}}{2 \Delta x}\left(\hat{\mathbf{R}}_{i+1}^{l}-2 \hat{\mathbf{R}}_{i}^{l}+\hat{\mathbf{R}}_{i-1}^{l}\right) \\
& -\frac{v \phi^{1 / 2}}{4}\left(\gamma_{i}^{l}-\gamma_{i-1}^{l}+\boldsymbol{\beta}_{i+1}^{l}-\boldsymbol{\beta}_{i}^{l}\right)-\frac{\mu}{3} \frac{\mathbf{B}\left(\hat{\mathbf{\Theta}}_{i+1}^{l}\right)-2 \mathbf{B}\left(\hat{\mathbf{\Theta}}_{i}^{l}\right)+\mathbf{B}\left(\hat{\mathbf{\Theta}}_{i-1}^{l}\right)}{\Delta x^{2}}, \\
& \boldsymbol{\xi}_{2}\left(\hat{\mathbf{R}}_{i}^{l}, \hat{\boldsymbol{\Theta}}_{i}^{l}\right)=\frac{1}{\varepsilon^{2}}\left(\mathbf{B}\left(\hat{\boldsymbol{\Theta}}_{i}^{l}\right)-\hat{\mathbf{R}}_{i}^{l}\right)+\frac{\mu}{3} \frac{\mathbf{B}\left(\hat{\boldsymbol{\Theta}}_{i+1}^{l}\right)-2 \mathbf{B}\left(\hat{\boldsymbol{\Theta}}_{i}^{l}\right)+\mathbf{B}\left(\hat{\boldsymbol{\Theta}}_{i-1}^{l}\right)}{\Delta x^{2}},
\end{aligned}
$$

with $\boldsymbol{\gamma}_{i}^{l}$ and $\boldsymbol{\beta}_{i}^{l}$ the same as (28).

We omit the details of velocity discretization and other space discretization, which is the same as in section 2.3. On the left-hand side of (70), we approximate $\mathbf{C}(\hat{\mathbf{\Theta}})_{i+1 / 2}^{k-1}$ by a second order discretization

$$
\mathbf{C}(\hat{\boldsymbol{\Theta}})_{i+1 / 2}^{k-1} \approx \frac{1}{2}\left(\mathbf{C}(\hat{\boldsymbol{\Theta}})_{i+1}^{k-1}+\mathbf{C}(\hat{\boldsymbol{\Theta}})_{i}^{k-1}\right) .
$$

Notice that to obtain $\hat{\boldsymbol{\Theta}}_{i}^{k}$, one needs to invert a large $K\left(N_{x}+1\right) \times K\left(N_{x}+1\right)$ matrix $\mathbf{M}$, where $N_{x}+1$ is the number of discretized points in space. $\mathbf{M}$ is defined as follows:

$$
\mathbf{M}_{K i+1: K(i+1), K j+1: K(j+1)}=\left\{\begin{array}{ll}
\mathbf{m}_{i}^{1} & \text { for } j=i-1, \\
\mathbf{m}_{i}^{2} & \text { for } j=i, \\
\mathbf{m}_{i}^{3} & \text { for } j=i+1, \\
0 & \text { otherwise, }
\end{array}, \quad i=0,1, \ldots, N_{x},\right.
$$

with $K \times K$ matrix $\mathbf{m}^{1}, \mathbf{m}^{2}$, and $\mathbf{m}^{3}$ :

$$
\begin{aligned}
\mathbf{m}_{i}^{1}= & -\frac{\Delta t a_{k k}}{\Delta x^{2}}\left(1+\frac{\Delta t a_{k k}}{\varepsilon^{2}}\right) \mathbf{I}-\frac{\left(\Delta t a_{k k}\right)^{2}}{2 \varepsilon^{2} \Delta x^{2}} \frac{4 \mu}{3}\left[\mathbf{C}(\hat{\boldsymbol{\Theta}})_{i-1}^{k-1}+\mathbf{C}(\hat{\boldsymbol{\Theta}})_{i}^{k-1}\right] ; \\
\mathbf{m}_{i}^{2}= & \left(1+\frac{\Delta t a_{k k}}{\varepsilon^{2}}\right) \mathbf{I}+\frac{\Delta t a_{k k}}{\varepsilon^{2}} 4 \mathbf{C}(\hat{\boldsymbol{\Theta}})_{i}^{k-1}+\frac{2 \Delta t a_{k k}}{\Delta x^{2}}\left(1+\frac{\Delta t a_{k k}}{\varepsilon^{2}}\right) \mathbf{I} \\
& +\frac{\left(\Delta t a_{k k}\right)^{2}}{2 \varepsilon^{2} \Delta x^{2}} \frac{4 \mu}{3}\left[\mathbf{C}(\hat{\boldsymbol{\Theta}})_{i+1}^{k-1}+2 \mathbf{C}(\hat{\boldsymbol{\Theta}})_{i}^{k-1}+\mathbf{C}(\hat{\boldsymbol{\Theta}})_{i-1}^{k-1}\right] ; \\
\mathbf{m}_{i}^{3}= & -\frac{\Delta t a_{k k}}{\Delta x^{2}}\left(1+\frac{\Delta t a_{k k}}{\varepsilon^{2}}\right) \mathbf{I}-\frac{\left(\Delta t a_{k k}\right)^{2}}{2 \varepsilon^{2} \Delta x^{2}} \frac{4 \mu}{3}\left[\mathbf{C}(\hat{\boldsymbol{\Theta}})_{i}^{k-1}+\mathbf{C}(\hat{\boldsymbol{\Theta}})_{i+1}^{k-1}\right] .
\end{aligned}
$$

From [19], one can prove the matrix $\left(1+\frac{\Delta t a_{k k}}{\varepsilon^{2}}\right) \mathbf{I}+\frac{\Delta t a_{k k}}{\varepsilon^{2}} 4 \mathbf{C}(\hat{\mathbf{\Theta}})_{i}^{k-1}$ is symmetric and positive definite under some gentle conditions. Then obviously, $\mathbf{M}$ is strictly block diagonal dominant, thus invertible. Since $\mathbf{M}$ is also sparse, a fast algorithm can be applied to solve for $\hat{\boldsymbol{\Theta}}^{k}$, then $\hat{\mathbf{R}}^{k}$ and $\hat{\mathbf{J}}^{k}$ can be obtained from (64a) and (64b) subsequently. 
3.4. The sAP property. Similarly as in section 2.4 , setting $\varepsilon \rightarrow 0$ in (64), one gets

$$
\begin{aligned}
& \hat{\mathbf{R}}_{i}^{k}=\mathbf{B}\left(\hat{\mathbf{\Theta}}_{i}^{k}\right), \\
& \hat{\mathbf{J}}_{i}^{k}=-v \frac{\hat{\mathbf{R}}_{i+1}^{k}-\hat{\mathbf{R}}_{i-1}^{k}}{2 \Delta x}, \\
& \left\langle\hat{\mathbf{R}}_{i}^{k}\right\rangle=\mathbf{B}\left(\hat{\Theta}_{i}^{k}\right) .
\end{aligned}
$$

$$
\hat{\mathbf{r}}_{i}^{n+1}+\hat{\boldsymbol{\theta}}_{i}^{n+1}=\hat{\mathbf{r}}_{i}^{n}+\hat{\boldsymbol{\theta}}_{i}^{n}+\Delta t \sum_{k=1}^{s} \tilde{b}_{k} \hat{\boldsymbol{\xi}}_{1}\left(\hat{\mathbf{R}}_{i}^{k}, \hat{\boldsymbol{\Theta}}_{i}^{k}\right)+\Delta t \sum_{k=1}^{s} b_{k} \hat{\boldsymbol{\xi}}_{2}\left(\hat{\mathbf{R}}_{i}^{k}, \hat{\boldsymbol{\Theta}}_{i}^{k}\right)+\Delta t \sum_{k=1}^{s} b_{k} \hat{h}\left(\hat{\boldsymbol{\Theta}}_{i}^{k}\right),
$$

where

$$
\begin{aligned}
& \hat{\boldsymbol{\xi}_{1}}\left(\hat{\mathbf{R}}^{k}, \hat{\boldsymbol{\Theta}}^{k}\right)=v^{2} \frac{\hat{\mathbf{R}}_{i+2}^{k}-2 \hat{\mathbf{R}}_{i}^{k}+\hat{\mathbf{R}}_{i-2}^{k}}{4 \Delta x^{2}}-\frac{\mathbf{B}\left(\hat{\boldsymbol{\Theta}}_{i+1}^{k}\right)-2 \mathbf{B}\left(\hat{\boldsymbol{\Theta}}_{i}^{k}\right)+\mathbf{B}\left(\hat{\boldsymbol{\Theta}}_{i-1}^{k}\right)}{3 \Delta x^{2}}, \\
& \hat{\boldsymbol{\xi}_{2}}\left(\hat{\boldsymbol{\Theta}}^{k}\right)=\frac{\mathbf{B}\left(\hat{\boldsymbol{\Theta}}_{i+1}^{k}\right)-2 \mathbf{B}\left(\hat{\boldsymbol{\Theta}}_{i}^{k}\right)+\mathbf{B}\left(\hat{\boldsymbol{\Theta}}_{i-1}^{k}\right)}{3 \Delta x^{2}}, \\
& \hat{h}\left(\hat{\boldsymbol{\Theta}}^{k}\right)=\frac{\hat{\boldsymbol{\Theta}}_{i+1}^{k}-2 \hat{\boldsymbol{\Theta}}_{i}^{k}+\hat{\boldsymbol{\Theta}}_{i-1}^{k}}{\Delta x^{2}} .
\end{aligned}
$$

Take $\langle\cdot\rangle$ on (74) and use (73c),

$$
\begin{aligned}
\hat{\boldsymbol{\theta}}_{i}^{n+1}+\mathbf{B}\left(\hat{\boldsymbol{\theta}}_{i}^{n+1}\right)= & \hat{\boldsymbol{\theta}}_{i}^{n}+\mathbf{B}\left(\hat{\boldsymbol{\theta}}_{i}^{n}\right)+\Delta t \sum_{k=1}^{s} b_{k}\left[\frac{\hat{\boldsymbol{\Theta}}_{i+1}^{k}-2 \hat{\boldsymbol{\Theta}}_{i}^{k}+\hat{\boldsymbol{\Theta}}_{i-1}^{k}}{\Delta x^{2}}\right. \\
& \left.+\frac{\mathbf{B}\left(\hat{\boldsymbol{\Theta}}_{i+1}^{k}\right)-2 \mathbf{B}\left(\hat{\boldsymbol{\Theta}}_{i}^{k}\right)+\mathbf{B}\left(\hat{\boldsymbol{\Theta}}_{i-1}^{k}\right)}{3 \Delta x^{2}}\right]+O\left(\Delta x^{2}\right),
\end{aligned}
$$

where

$$
\begin{aligned}
\hat{\boldsymbol{\Theta}}_{i}^{k}+\mathbf{B}\left(\hat{\boldsymbol{\Theta}}_{i}^{k}\right)= & \hat{\boldsymbol{\theta}}_{i}^{n}+\mathbf{B}\left(\hat{\boldsymbol{\theta}}_{i}^{n}\right)+\Delta t \sum_{l=1}^{k} a_{k l}\left[\frac{\hat{\boldsymbol{\Theta}}_{i+1}^{l}-2 \hat{\boldsymbol{\Theta}}_{i}^{l}+\hat{\boldsymbol{\Theta}}_{i-1}^{l}}{\Delta x^{2}}\right. \\
& \left.+\frac{\mathbf{B}\left(\hat{\boldsymbol{\Theta}}_{i+1}^{l}\right)-2 \mathbf{B}\left(\hat{\boldsymbol{\Theta}}_{i}^{l}\right)+\mathbf{B}\left(\hat{\boldsymbol{\Theta}}_{i-1}^{l}\right)}{3 \Delta x^{2}}\right]
\end{aligned}
$$

which is an implicit RK method for the limiting diffusion equation (58). Thus the sAP property is demonstrated.

4. Numerical tests. In this section we present several numerical tests to show the performance of the new sAP IMEX schemes. We use the notation SSP2 to denote the new scheme based on the second order IMEX-RK tableau (24) and JPT to denote the second order scheme in [20] with the SG formulation. First we test the new approach in the deterministic case and then we consider the presence of uncertainties.

4.1. Test 1: The linear transport equation. We consider problem (1) with

$$
x \in[0,1], F_{L}(v)=1, F_{R}(v)=0, \sigma_{s}=1, \sigma_{A}=0, Q=0, \varepsilon=10^{-6} .
$$




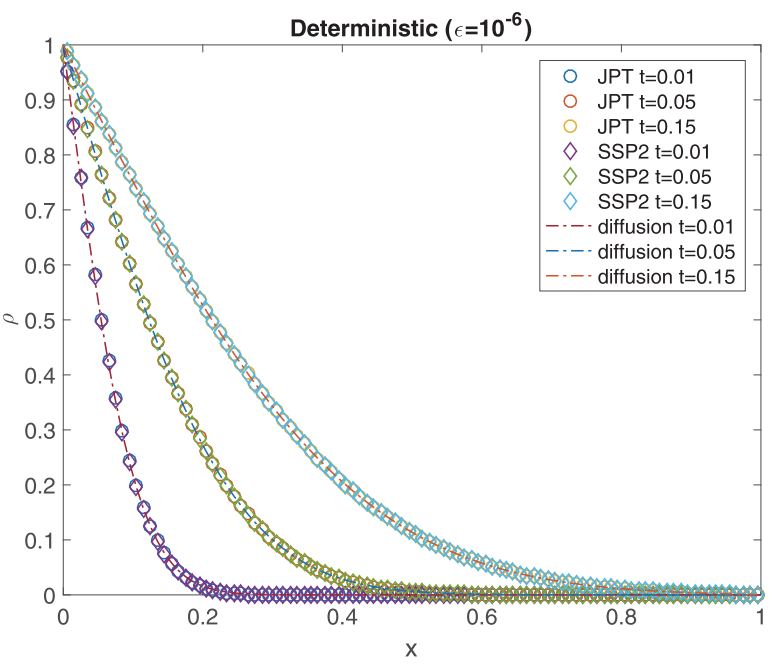

FIG. 1. Test 1. The solution of the mass density $\rho$ to the linear transport equation. JPT (०), $\Delta x=0.01, \Delta t=0.5(\Delta x)^{2}, N T=200,1000,3000 ;$ SSP2 $(\diamond), \Delta x=0.01, \Delta t=\lambda \Delta x$ with $\lambda=0.04$, $N T=25,125,375$.

TABLE 1

Test 1. Time cost comparison in seconds between JPT and SSP2 schemes.

\begin{tabular}{c|c|c|c}
\hline & $t=0.01$ & $t=0.05$ & $t=0.15$ \\
\hline JPT & $0.451567 \mathrm{~s}$ & $2.014770 \mathrm{~s}$ & $6.069805 \mathrm{~s}$ \\
\hline SSP2 & $0.069801 \mathrm{~s}$ & $0.290240 \mathrm{~s}$ & $0.815041 \mathrm{~s}$ \\
\hline
\end{tabular}

We report the results at different times $t=0.01, t=0.05$, and $t=0.15$ in Figure 1. In all figures we use the notation $N T$ to denote the number of time steps. With fixed $\Delta x=0.01$, for JPT (o) $\Delta t=0.5(\Delta x)^{2}, N T=200,1000,3000$; for SSP2 $(\diamond) \Delta t=\lambda \Delta x$ with $\lambda=0.04, N T=25,125,375$. The exact diffusive solution is computed by (2) with the same mesh size in the dashed line. One can see that both methods are AP and work well in the diffusive regime at any time. The time cost of the two methods is reported in Table 1, which shows that SSP2 is about 7 times more efficient than JPT.

4.2. Test 2: The linear transport equation with random inputs. We consider problem (1) with random inputs and

$$
x \in[0,1], F_{L}(v)=1, F_{R}(v)=0, \sigma_{s}=1+0.5 z, \sigma_{A}=0, Q=0, \varepsilon=10^{-6},
$$

where $z$ follows uniform distribution on $[-1,1]$ (denoted by $z \sim \mathcal{U}[-1,1]$ in the following tests).

4.2.1. Diffusive regime: $\varepsilon=10^{-6}$. The mean and standard deviation are two quantities of interest. So we examine these two quantities of the numerical solutions at different times $t=0.01, t=0.05$ and $t=0.15$ in Figure 2. Fourth order gPC basis $(K=5)$ is used here and after. Given the gPC coefficients $\hat{\rho}_{k}$ of $\rho$, it's convenient to calculate the mean value and standard deviation by

$$
\mathrm{E}[\rho] \approx \hat{\rho}_{1}, \operatorname{Sd}[\rho] \approx \sqrt{\sum_{k=2}^{K} \hat{\rho}_{k}^{2} .}
$$



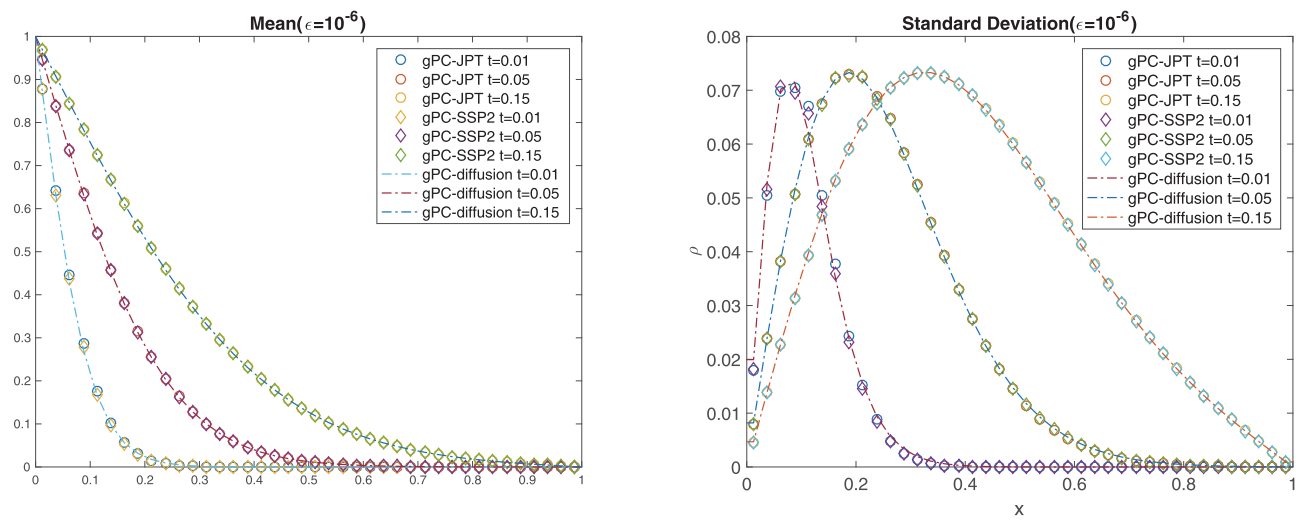

FIG. 2. Test 2. The mean (left) and standard deviation (right) of the solution of the mass density $\rho$ to the linear transport equation. JPT (०), $\Delta x=0.025, \Delta t=0.0002, N T=50,250,750$; $S S P 2(\diamond), \Delta x=0.025, \Delta t=\lambda \Delta x$ with $\lambda=0.035, N T=11,57,171$.
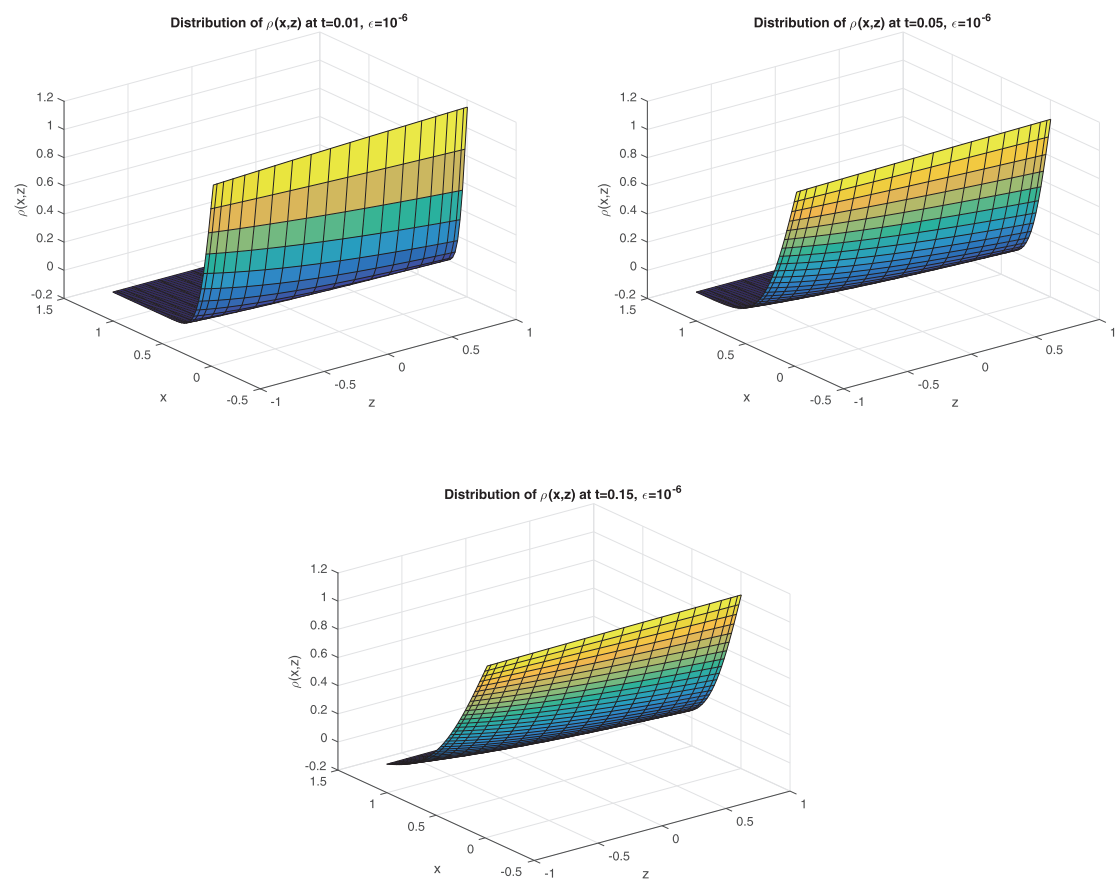

FIG. 3. Test 2. Mass density $\rho(x, z)$ of the linear transport equation by $g P C-S S P 2$ with $N T=$ $11,57,171$.

With fixed $\Delta x=0.025$, for JPT (o) $\Delta t=0.0002$ and $N T=50,250,750$; for SSP2 $(\diamond) \Delta t=\lambda \Delta x$ with $\lambda=0.035, N T=11,57,171$. The exact diffusive solution is represented by the dashed line. As expected, both methods are sAP and match well in the diffusive regime at any time. The distribution of $\rho(x, z)$ at different times is presented in Figure 3. We report the time cost comparison of gPC-JPT and gPC-SSP2 in Table 2, which shows that gPC-SSP2 is more than 3 times faster than gPC-JPT. Since randomness is introduced and computation becomes more time-consuming, the efficiency of gPC-SSP2 is remarkable. 
TABLE 2

Test 2. Time cost comparison in seconds between JPT and SSP2 schemes for $\varepsilon=10^{-6}$.

\begin{tabular}{c|c|c|c}
\hline & $t=0.01$ & $t=0.05$ & $t=0.15$ \\
\hline gPC-JPT & $2.662382 \mathrm{~s}$ & $13.159598 \mathrm{~s}$ & $39.255826 \mathrm{~s}$ \\
\hline gPC-SSP2 & $0.759259 \mathrm{~s}$ & $3.865332 \mathrm{~s}$ & $11.424931 \mathrm{~s}$ \\
\hline
\end{tabular}
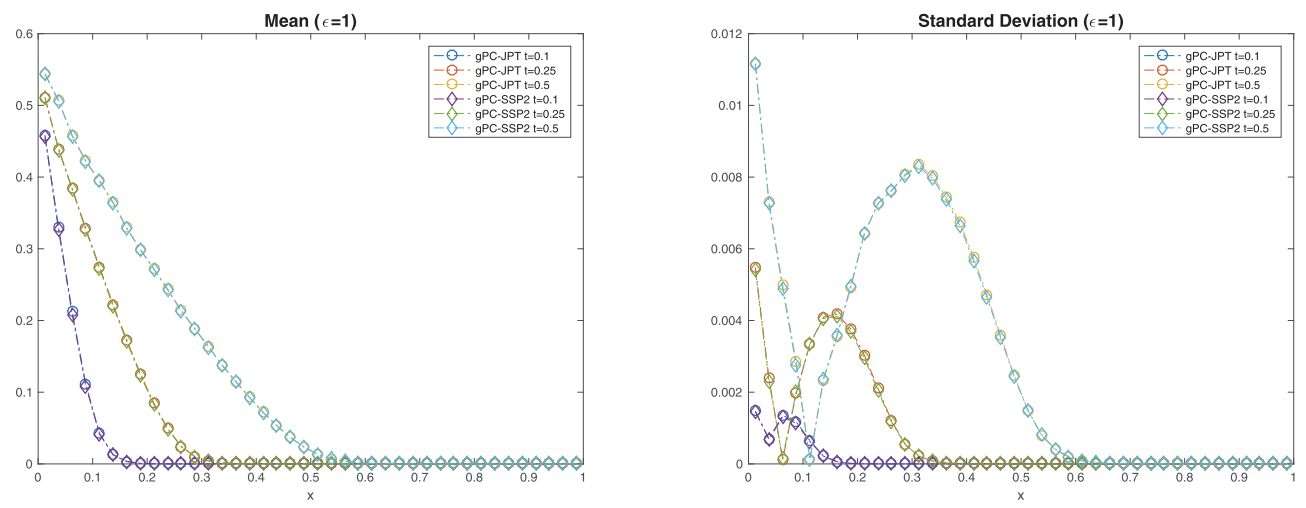

FIG. 4. Test 2. The mean (left) and standard deviation (right) of the solution of the mass density $\rho$ to the linear transport equation. JPT (०), $\Delta x=0.025, \Delta t=0.0002, N T=500,1250,2500$; $S S P 2(\diamond), \Delta x=0.025, \Delta t=\lambda \Delta x$ with $\lambda=0.035, N T=114,285,571$.

4.2.2. Kinetic regime: $\varepsilon=1$. Similarly as in the experiments in the diffusive regime, we examine the mean and standard deviation of the numerical solutions at different times $t=0.1, t=0.25$, and $t=0.5$ in Figure 4 . With the same mesh size and notation, we compare the gPC-JPT (o) and the gPC-SSP2 $(\diamond)$ methods and we can see they match well in the kinetic regime as expected. The distribution of $\rho(x, z)$ at different times is presented in Figure 5 and the time cost comparison is reported in Table 3 .

4.2.3. Convergence test. In this section we investigate the convergence rate of the gPC-SSP2 method numerically in the diffusive regime $\left(\varepsilon=10^{-6}\right)$ and the kinetic regime $(\varepsilon=1)$, respectively. Numerical results in [21] already showed the spectral convergence of the gPC approach. Thus here we refine the spatial mesh by 2 with fixed degree of gPC polynomials $K=8$. Relative error of mean and standard deviation until $t=0.1$ for $\mathrm{NX}=40,80,160,320$ in $L^{\infty}$ norm are plotted in log-log scale in Figure 6. The second order convergence is reached as expected.

4.3. Test 3: The radiative heat transfer equation. We then compare the two methods for deterministic radiative heat transfer equations (44) near diffusive regime $\left(\varepsilon=10^{-6}\right)$ with the initial and boundary conditions

$$
\begin{aligned}
& I_{I}(x, \mu, z, 0)=0, \quad \theta_{I}(x, z, 0)=0, \quad x \in[0,1], \\
& \theta_{B}(0, z, t)=1, \theta_{B}(1, z, t)=0 ; \\
& I_{B}(0, \mu, z, t)=1, \mu>0, \quad I_{B}(1, \mu, z, t)=0, \mu<0,
\end{aligned}
$$

and constant coefficient

$$
\sigma(x)=1 .
$$

Figure 7 shows the solutions of temperature $\theta$ at different times $t=0.01, t=0.05$, and $t=0.15$. With fixed $\Delta x=0.025$, for JPT (॰) $\Delta t=0.002$; for SSP2 ( $\diamond) \Delta t=\lambda \Delta x$ 

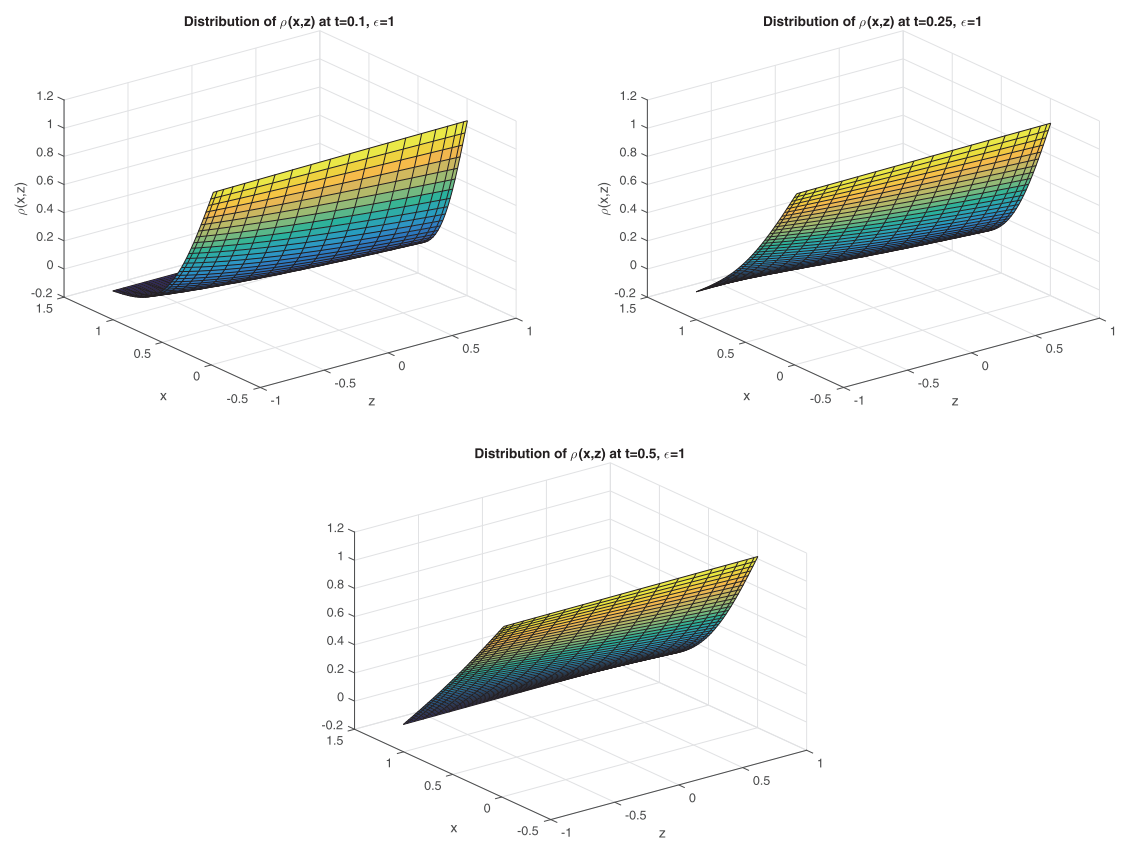

FIG. 5. Test 2. Mass density $\rho(x, z)$ of the linear transport equation by $g P C-S S P 2$ with $N T=$ $114,285,571$

TABLE 3

Test 2. Time cost comparison in seconds between JPT and SSP2 schemes for $\varepsilon=1$.

\begin{tabular}{c|c|c|c}
\hline & $t=0.1$ & $t=0.25$ & $t=0.5$ \\
\hline gPC-JPT & $21.332570 \mathrm{~s}$ & $66.430655 \mathrm{~s}$ & $114.492939 \mathrm{~s}$ \\
\hline gPC-SSP2 & 11.232046 & $30.808011 \mathrm{~s}$ & $81.000325 \mathrm{~s}$ \\
\hline
\end{tabular}

with $\lambda=0.035$. The exact diffusion solution is computed by (54) with the same mesh size in the dashed line. One can see that both methods are AP and work well in the diffusive regime at any time. The time cost of both methods is reported in Table 4, which shows that SSP2 is about two times faster than JPT in this nonlinear case.

4.4. Test 4: The radiative heat transfer equation with random inputs. We then compare the two methods in (44) with randomness in the cross section with the initial and boundary conditions

$$
\begin{aligned}
& I_{I}(x, \mu, z, 0)=0, \quad \theta_{I}(x, z, 0)=0, \quad x \in[0,1], \\
& \theta_{B}(0, z, t)=1, \theta_{B}(1, z, t)=0 ; \\
& I_{B}(0, \mu, z, t)=1+0.5 z, \mu>0, \quad I_{B}(1, \mu, z, t)=0, \mu<0,
\end{aligned}
$$

and random coefficient

$$
\sigma(z)=1+0.5 z, \quad z \sim \mathcal{U}[-1,1]
$$

In Figure 8, we report the mean and standard deviation of temperature $\theta$ at different times $t=0.01, t=0.05$, and $t=0.15$. Similar to Test 2 , the mean value and standard deviation are calculated by 

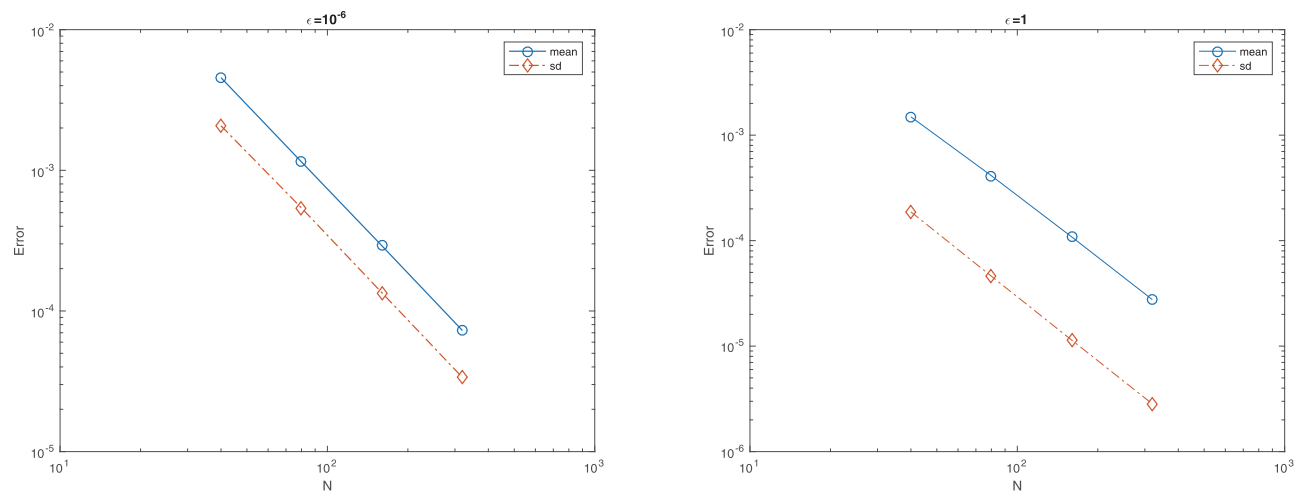

FIG. 6. Test 2. Errors in mean and standard deviation versus $N$ of gPC-SSP2 method in diffusive regime $\left(\varepsilon=10^{-6}\right)$ and kinetic regime $(\varepsilon=1)$.

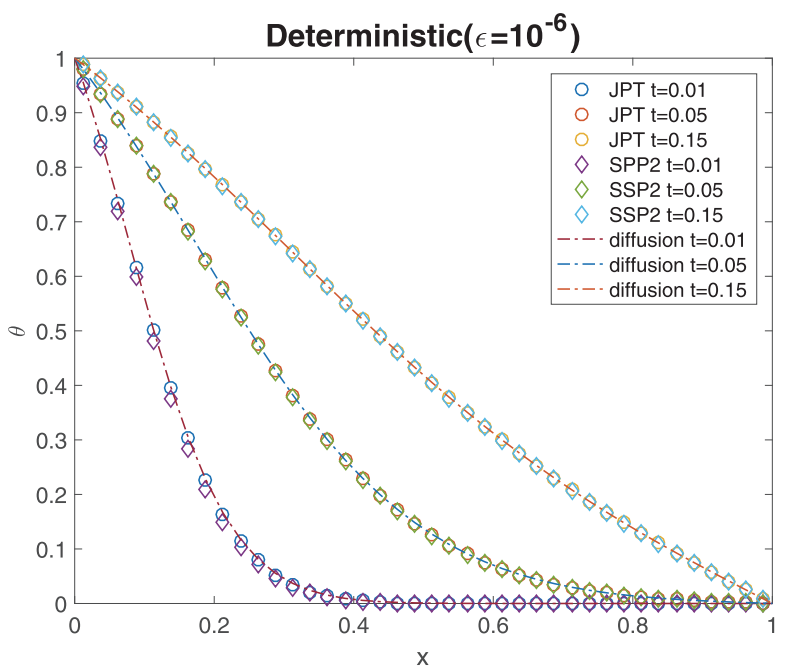

FIG. 7. Test 3. The solution of temperature $\theta$ to the radiative heat transfer equation. JPT (o), $\Delta x=0.025, \Delta t=0.0002, N T=50,250,750 ; S S P 2(\diamond), \Delta x=0.025, \Delta t=\lambda \Delta x$ with $\lambda=0.035$, $N T=11,57,171$.

TABLE 4

Test 3. Time cost comparison in seconds between JPT and SSP2 schemes.

\begin{tabular}{c|c|c|c}
\hline & $t=0.01$ & $t=0.05$ & $t=0.15$ \\
\hline JPT & $0.112507 \mathrm{~s}$ & $0.268415 \mathrm{~s}$ & $0.806410 \mathrm{~s}$ \\
\hline SSP2 & $0.059658 \mathrm{~s}$ & $0.208812 \mathrm{~s}$ & $0.583166 \mathrm{~s}$ \\
\hline
\end{tabular}

$$
\mathrm{E}[\theta] \approx \hat{\theta}_{1}, \operatorname{Sd}[\theta] \approx \sqrt{\sum_{k=2}^{K} \hat{\theta}_{k}^{2}} .
$$

With fixed $\Delta x=0.025$, for JPT (०) $\Delta t=0.0002$ and $N T=50,250,750$; for SSP2 $(\diamond) \Delta t=\lambda \Delta x$ with $\lambda=0.035, N T=11,57,171$. The reference diffusion solution is represented by the dashed line. We refer to [19] for the same results using the 

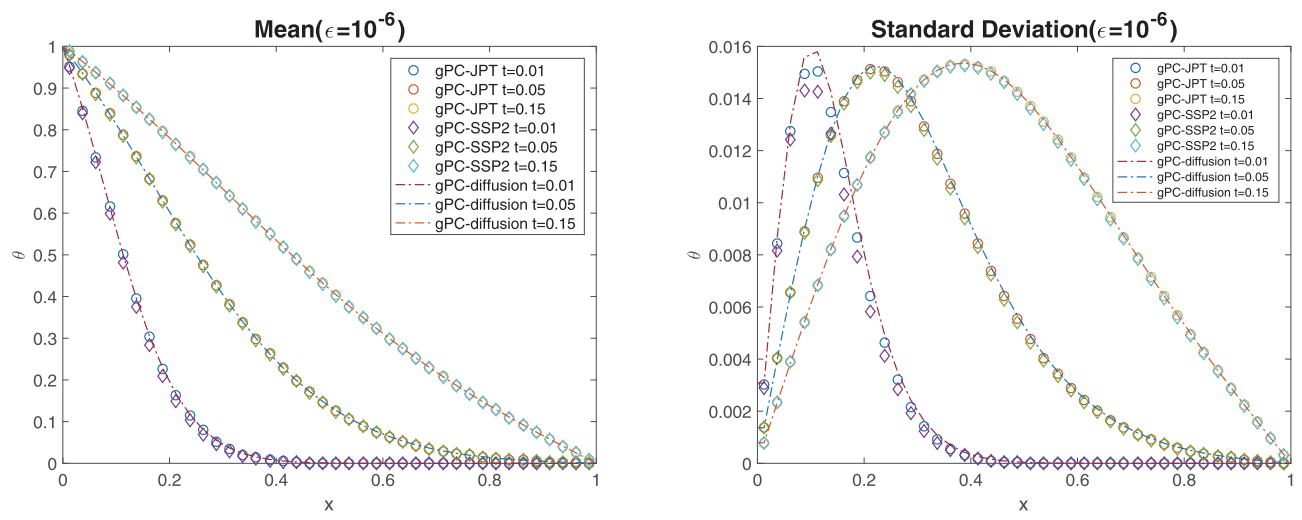

FIG. 8. Test 4. The mean (left) and standard deviation (right) of the solution of temperature $\theta$ to the radiative heat transfer equation. JPT (०), $\Delta x=0.025, \Delta t=0.0002, N T=50,250,750$; $\operatorname{SSP2}(\diamond), \Delta x=0.025, \Delta t=\lambda \Delta x$ with $\lambda=0.035, N T=11,57,171$.
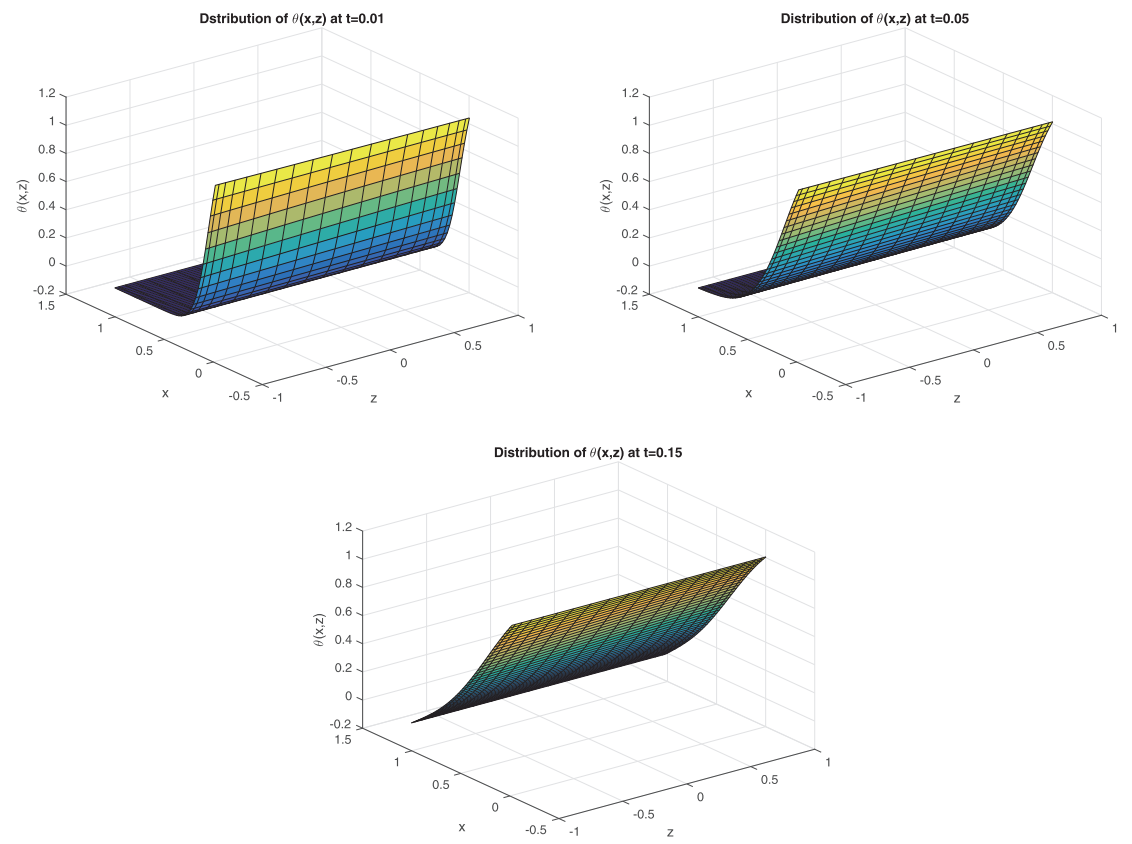

FIG. 9. Test 2. Temperature $\theta(x, z)$ of the radiative heat transfer equation by $g P C-S S P 2$ with $N T=11,57,171$.

micro-macro decomposition based gPC method. Good agreements of the gPC-JPT and gPC-SSP2 methods and the reference solution are observed in the diffusive regime at plotted times. The distribution of $\theta(x, z)$ at different times is depicted in Figure 9. We report the time cost comparison of gPC-JPT and gPC-SSP2 in Table 5, which shows that gPC-SSP2 is nearly 4 times faster than gPC-JPT. The nonlinearity slows down gPC-JPT a lot and thus the advantage in efficiency of gPC-SSP2 is significant in this nonlinear problem with random inputs. 
TABLE 5

Test 4. Time cost comparison in seconds between JPT and SSP2 schemes.

\begin{tabular}{c|c|c|c}
\hline & $t=0.01$ & $t=0.05$ & $t=0.15$ \\
\hline gPC-JPT & $172.431508 \mathrm{~s}$ & $804.915170 \mathrm{~s}$ & $2329.405654 \mathrm{~s}$ \\
\hline gPC-SSP2 & $36.945166 \mathrm{~s}$ & $192.040137 \mathrm{~s}$ & $626.736282 \mathrm{~s}$ \\
\hline
\end{tabular}

5. Conclusions. In this article, for linear transport and radiative heat transfer equations with random inputs, new generalized polynomial chaos based asymptoticpreserving stochastic Galerkin schemes are introduced. Compared with previous methods for these problems, our new method uses the implicit-explicit time discretization to gain higher order accuracy, and by using a modified diffusion operator based penalty method, a more relaxed stability condition - a hyperbolic, rather than parabolic - CFL condition is achieved when the Knudsen number is small, in the diffusive regime.

These schemes allow efficient computation of random transport equations that contain both uncertainties and multiscales, allowing Knudsen number independent time step, mesh size, and degree of polynomials, with a spectral accuracy in the random space. They can be used efficiently for all ranges of Knudsen numbers.

There remain many issues to be resolved. First, we only studied the one-dimensional problems in space and velocity. The next step will be to extend the methods to higher dimensions. In addition, a multidimensional challenge is also presented in the random space. Sparse grids could be used, as was done in [32], but it remains to be explored.

\section{REFERENCES}

[1] C. Bardos, R. Santos, And R. Sentis, Diffusion approximation and computation of the critical size, Trans. Amer. Math. Soc., 284 (1984), pp. 617-649.

[2] S. Boscarino, L. Pareschi, and G. Russo, Implicit-explicit Runge-Kutta schemes for hyperbolic systems and kinetic equations in the diffusion limit, SIAM J. Sci. Comput., 35 (2013), pp. A22-A51.

[3] S. Boscarino, L. Pareschi, And G. Russo, A unified IMEX Runge-Kutta approach for hyperbolic systems with multiscale relaxation, SIAM J. Numer. Anal., 55 (2017), pp. 2085-2109.

[4] F. Bouchut, F. Golse, And M. Pulvirenti, Kinetic Equations and Asymptotic Theory, Ser. Appl. Math., B. Perthame and L. Desvillettes, eds., Elsevier, New York, 2000.

[5] C. BuET AND S. CoRdier, An asymptotic preserving scheme for hydrodynamics radiative transfer models, Numer. Math., 108 (2007), pp. 199-221.

[6] J. A. Carrillo, T. Goudon, P. Lafitte, and F. Vecil, Numerical schemes of diffusion asymptotics and moment closures for kinetic equations, J. Sci. Comput., 36 (2008), pp. 113-149.

[7] Z. ChEn, L. LIU, AND L. MU, DG-IMEX stochastic Galerkin schemes for linear transport equation with random inputs and diffusive scalings, J. Sci. Comput., 73 (2017), pp. 1-27.

[8] K. M. Case and P. F. Zweifel, Linear Transport Theory, Addison-Wesley, Reading, MA, 1997.

[9] F. Cavalli, G. Naldi, G. Puppo, and M. Semplice, High order relaxation schemes for non linear diffusion problems, SIAM J. Numer. Anal., 45 (2007), pp. 2098-2119.

[10] S. Chandrasekhar, Radiative Transport, Dover, New York, 1960.

[11] P. Degond, Asymptotic-Preserving Schemes for Fluid Models of Plasmas, Numerical Models for Fusion, Panor. Syntheses 39/40, Société Mathématique, France, Paris, 2013, pp. 1-90.

[12] P. Degond and F. Deluzet, Asymptotic-preserving methods and multiscale models for plasma physics, J. Comput. Phys., 336 (2017), pp. 429-457.

[13] G. Dimarco and L. Pareschi, Numerical methods for kinetic equations, Acta Numer., 23 (2014), pp. 369-520.

[14] L. Gosse And G. Toscani, Asymptotic-preserving and well-balanced schemes for radiative transfer and the Rosseland approximation, Numer. Math., 98 (2004), pp. 223-250. 
[15] J. Hu And S. Jin, A stochastic Galerkin method for the Boltzmann equation with uncertainty, J. Comput. Phys., 315 (2016), pp. 150-168.

[16] S. Jin, Asymptotic Preserving (AP) Schemes for Multiscale Kinetic and Hyperbolic Equations: A Review, Lecture notes, Porto Ercole, Grosseto, Italy, 2010, pp. 177-216.

[17] S. Jin, J.-G. LIU, AND Z. MA, Uniform spectral convergence of the stochastic Galerkin method for the linear transport equations with random inputs in diffusive regime and a micromacro decomposition based asymptotic preserving method, Res. Math. Sci., 4 (2017).

[18] S. Jin AND L. LiU, An asymptotic-preserving stochastic galerkin method for the semiconductor Boltzmann equation with random inputs and diffusive scalings, Multiscale Model. Simul., 15 (2017), pp. 157-183.

[19] S. Jin AND H. LU, An asymptotic-preserving stochastic Galerkin method for the radiative heat transfer equations with random inputs and diffusive scalings, J. Comput. Phys., 334 (2017), pp. 182-206.

[20] S. Jin, L. PAReschi, AND G. Toscani, Uniformly accurate diffusive relaxation schemes for multiscale transport equations, SIAM J. Numer. Anal., 38 (2000), pp. 913-936.

[21] S. Jin, D. XIU, AND X. ZHU, Asymptotic-preserving methods for hyperbolic and transport equations with random inputs and diffusive scalings, J. Comput. Phys., 289 (2015), pp. $35-52$.

[22] A. KLAR, An asymptotic-induced scheme for nonstationary transport equations in the diffusive limit, SIAM J. Numer. Anal., 35 (1998), pp. 1073-1094.

[23] A. Klar and C. Schmeiser, Numerical passage from radiative heat transfer to nonlinear diffusion models, Math. Models Methods Appl. Sci., 11 (2001), pp. 749-767.

[24] P. Lafitte and G. Samaey, Asymptotic-preserving projective integration schemes for kinetic equations in the diffusion limit, SIAM J. Sci. Comput., 34 (2012), pp. A579-A602.

[25] E. W. Larsen and J. B. Keller, Asymptotic solution of neutron transport problems for small mean free paths, J. Math. Phys., 15 (1974), pp. 75-81.

[26] E. W. Larsen and J. E. Morel, Asymptotic solutions of numerical transport problems in optically thick, diffusive regimes II, J. Comput. Phys., 83 (1989), pp. 212-236.

[27] E. W. Larsen, J. E. Morel, and W. F. Miller, Jr., Asymptotic solutions of numerical transport problems in optically thick, diffusive regimes, J. Comput. Phys., 69 (1987), pp. $283-324$.

[28] M. Lemou And L. Mieussens, A new asymptotic preserving scheme based on micro-macro formulation for linear kinetic equations in the diffusion limit, SIAM J. Sci. Comput., 31 (2010), pp. 334-368.

[29] Q. Li AND L. WANG, Uniform regularity for linear kinetic equations with random input based on hypocoercivity, SIAM/ASA J. Uncertain. Quantif., 5 (2017), pp. 1193-1219.

[30] G. NAldi AND L. PARESCHI, Numerical schemes for hyperbolic systems of conservation laws with stiff diffusive relaxation, SIAM J. Numer. Anal., 37 (2000), pp. 1246-1270.

[31] L. PARESChI AND G. Russo, Implicit-Explicit Runge-Kutta schemes and applications to hyperbolic systems with relaxations, J. Sci. Comput., 25 (2005), pp. 129-155.

[32] R. Shu AND S. Jin, A stochastic Asymptotic-Preserving scheme for a kinetic-fluid model for disperse two-phase flows with uncertainty, J. Comput. Phys., 335 (2017), pp. 905-924.

[33] H. Spohn, Large Scale Dynamics of Interacting Particles, Springer-Verlag, Berlin, 1991.

[34] W. Sun, S. JiAng, AND K. Xu, An asymptotic preserving unified gas kinetic scheme for gray radiative transfer equations, J. Comput. Phys., 285 (2015), pp. 265-279.

[35] D. XIU AND G.E. KARNIADAKIS, The Wiener-Askey polynomial chaos for stochastic differential equations, SIAM J. Sci. Comput., 24 (2002), pp. 619-644.

[36] Y. ZHU AND S. Jin, The Vlasov-Poisson-Fokker-Planck system with uncertainty and a onedimensional asymptotic-preserving method, SIAM Multiscale Model. Simul., 15 (2017), pp. $1502-1529$. 